\title{
Bubbles to Chondrites-II. Chemical fractionations in chondrites
}

\author{
Akihiko Hashimoto $^{1}$ (D) and Yuki Nakano ${ }^{2 *}$ (D)
}

\begin{abstract}
We attempt to develop a possible theory of chemical fractionations in chondrites, that is consistent with various features of chondritic components and current observation of protoplanetary disks (PPD). Combining the $3+2$ component fitting calculation that simulates chondrule formation process proposed in paper (I) with additional mixing procedures, we investigate essential causes that made various types of chondrites evolve from the uniform solar system composition, the $\mathrm{Cl}$-chondritic composition. Seven chemical types of chondrites (CM, CV, CO, E, LL, L and $\mathrm{H}$ ) are examined, for which reliable chemical compositions for both bulk chondrites and chondrules therein are known. High vaporization degree of the primordial dust aggregates (dustons) required by the calculation vindicates that the chondrule formation was the driving force for the chemical fractionations in all chondrites examined. Various initial redox states in dustons and different timings of CAls' invasion to the chondrule formation zone are identified for different chondrite types. These results, together with a good correlation with the $\mathrm{D} / \mathrm{H}$ ratios of chondrites measured previously, lead us to the notion that PPD evolved from reducing to oxidizing. We explore the heating mechanism for the chondrule formation and the place it occurred. Only heat source being consistent with our chondrule formation model is lightning discharge. We postulate that large vortices encompassing the snow-line are ideal places for large charge separation to occur between dustons and small ice particles, and that direct strikes on dustons should make them boil for ten seconds and longer and allow a swarm of chondrules released from their surfaces. Chemical fractionations are completed by an aerodynamic separation of dustons from chondrules inside the vortex, in such a way that the dustons fall fast into the vortex center and form a planetesimal immediately, while chondrules with dust mantles fall slow and form a thin veneer on the planetesimal surface. During collisional episodes, the veneers are preferentially fragmented and reassemble themselves by a weak self-gravity to form a rubble-piled chondritic asteroid, i.e. chondrite.
\end{abstract}

Keywords: Chondrites, Chondrules, Chemical fractionations, Protoplanetary disk, CAls, D/H ratio, Lightning discharge, Planetesimal, ${ }^{26} \mathrm{Al}$, Redox state

\section{Introduction}

In pursuit of fundamental rules of chemical fractionations caused by evaporation and condensation of primordial dust aggregates, we have discovered an alternative and compelling route in evolution of chondritic materials in the solar system protoplanetary disk.

From late 1960's to early 1980's, Anders and his group sent out an outstanding array of their research works

\footnotetext{
*Correspondence: yudaruma@ep.sci.hokudai.ac.jp

${ }^{2}$ Department of Cosmosciences, Hokkaido University, N10 W8, 060-0810,

Sapporo, Japan
}

Full list of author information is available at the end of the article on meteorites (e.g., a series of 'Chemical fractionations in meteorites'; Anders 1971), particularly on chondrites, deciphered a wealth of chemical codes about the origin of our solar system materials, and established an idea that chondritic materials are building-blocks of the planets. Their core idea, the equilibrium condensation of a hot and dense gas, initially above $2000 \mathrm{~K}$, with the solar elemental abundance, was inspired by Cameron's solar nebula model (Cameron 1962; 1963).

Recent theories and observations indicate much colder protoplanetary disks (PPD) with much smaller masses, as Williams and Cieza (2011) in their review paper described,
SpringerOpen

(c) The Author(s). 2021 Open Access This article is licensed under a Creative Commons Attribution 4.0 International License, which permits use, sharing, adaptation, distribution and reproduction in any medium or format, as long as you give appropriate credit to the original author(s) and the source, provide a link to the Creative Commons licence, and indicate if changes were made. The images or other third party material in this article are included in the article's Creative Commons licence, unless indicated otherwise in a credit line to the material. If material is not included in the article's Creative Commons licence and your intended use is not permitted by statutory regulation or exceeds the permitted use, you will need to obtain permission directly from the copyright holder. To view a copy of this licence, visit http://creativecommons.org/licenses/by/4.0/. 
"Initially, disks rapidly funnel material onto the star but, as the surrounding molecular core is used up or otherwise disperses, the accretion rate decreases and a small amount of material persists. That these discs can be considered protoplanetary is apparent through not only the geometry of the Solar System but also the high detection rate of exoplanets". On the other hand, accretion during the protostellar phase, while the protostellar envelope is still falling onto the disk, is much less well understood (Hartmann et al. 2016), and thus a theoretical possibility may exist that the planet- and meteorite-forming materials had gone through a high temperature phase when all the preexisting dust once became a gas.

This possibility, however, is denied because the meteorites retain presolar grains with large isotopic anomalies intact (e.g., Zinner 1998) and because the $\mathrm{D} / \mathrm{H}$ ratios of chondritic water and organic compounds exhibit $\sim 10^{-4}$ or higher values (e.g., Alexander et al. 2012) as opposed to the solar value $\sim 2 \times 10^{-5}$, which should be homogenized to the solar value if heated above $500 \mathrm{~K}$ (Lécluse and Robert 1994). Therefore, the equilibrium condensation model of chondritic materials has lost its basis and we are in need of a new mechanism to explain the chemical fractionations in chondrites.

Chondrules, once molten silicate droplets of $0.1-3 \mathrm{~mm}$ in size that constitute more than $60 \%$ of the chondrites in volume, have been generally regarded as nothing but a simple melted product of preexisting dust (e.g., Larimer and Anders 1970; Jacquet 2014), and only their heat sources are a matter of concern for most researchers. However, the chondrule formation event is only opportunity for causing chemical fractionations in chondrites because the idea of the nebular-scaled, high temperature phase is no longer valid.

In the companion paper (I) (Nakano and Hashimoto 2020), we have demonstrated that primordial dust aggregates that are dense and large ( $>3 \mathrm{~cm}$ in diameter), named dustons, are needed to generate jet-droplets, viz., chondrule nuclei, upon bursting of boiling bubbles on their surfaces. We have developed a model that a vapor cloud that forms around a boiling duston provides the environment where jet-droplets react with vapor and condensed particles on their way out and complete their chemical integrity as chondrules. This new model of chondrule formation explains the 3.5 fundamental features of chondrules, including their chemistry, size range and frequency distribution, and textures.

Here we extend our model to cover the bulk chemistry of chondrites and propose a new mechanism of chemical fractionations in chondrites starting with the chemical composition of CI chondrite.

This paper starts as a sequel to paper (I), in that basically the same model and calculation procedure are applied to the bulk chemistry of chondrites, but having gained an entirely new perspective on the origin of chondrites, we expand our view to the evolution of PPD materials from dust to planetesimals. We also investigate a possible heat source of chondrule formation that should be consistent with our model.

\section{Numerical model of chemical fractionation}

In our chondrite formation model, we assume the initial material to be primordial dust aggregate having a CI-like composition, $\mathrm{CI}^{\text {sil }}$, just as in our paper (I). $\mathrm{CI}^{\text {sil }}$ is the silicate composition made of the ten oxide elements in CIchondritic proportions, $\mathrm{Na}, \mathrm{Mg}, \mathrm{Al}, \mathrm{Si}, \mathrm{K}, \mathrm{Ca}, \mathrm{Ti}, \mathrm{Cr}, \mathrm{Mn}$ and $\mathrm{Fe}$, only controlled with a parameter $f_{s}$ that is a fraction of Fe oxide in the total Fe in CI. We do this, since Fe can also exist in other redox states such as metal and sulfide (See the detail in paper I).

In our paper (I), as a consequence of jet-droplet ejection in the vapor cloud that enshrouds the duston, we have recognized the three components: jet-droplets, vapor in the cloud (named differential vapor), and fine condensed particles (named integrated vapor). We have modeled chondrule chemistry as a mixture of the three, and successfully reproduced chemical compositions of $75 \%$ of the 600 real chondrules. We extend the same method with two more components, on account of the matrix material that coexists with chondrules inside chondrites.

\subsection{Methods}

We wish to solve the five simultaneous linear equations just as in paper (I),

$$
A_{m, n} F_{n}=B_{m}(m=1-5 \text { and } n=1-5) .
$$

Here $B_{m}$ represents a molar fraction of element $m=1-4$ $(\mathrm{Na}, \mathrm{Mg}, \mathrm{Al}$ and $\mathrm{Si}$ in ascending order) in a 'bulk silicate chondrite.' $B_{5}=1$. $F_{n}$, an unknown to be determined, represents a mixing proportion (in molar basis) of the five components $n=1-5$ (1: jet-droplet, 2: differential vapor, 3: integrated vapor, 4: final integrated vapor and 5: $\mathrm{CI}^{*}$ ). By definition $\sum_{n} F_{n}=1 . A_{m, n}$ is a matrix to describe molar fractions of $\mathrm{Na}, \mathrm{Mg}, \mathrm{Al}$ and $\mathrm{Si}(m=1-4)$ among the ten elements in each of the five components $(n=1-5)$. $A_{5, n}=1(n=1-5)$.

The three components $(n=1-3)$ are for reproducing a chemical composition of individual chondrules in paper (I). This time they are for an average composition of as many chondrules as available from a single chondrite or from a specified type of chondrites.

The two components ( $n=4$ and 5) are for making the matrix material in that single chondrite or in that specific type of chondrites. The fourth component $(n=4)$ represents the integrated vapor composition (viz., a weighted average composition of the total condensed particles) at the end of evaporation from 
the duston. For simplicity, the vapor lost (both differential and integrated) by reaction with jet-droplets is assumed minimal or zero. The termination of evaporation is signaled with the final VD (vaporization degree) reached by MLB (mother liquid body, viz., melted surface) of the duston, which is made equal to twice the VD that produces the average chondrule composition. It presupposes that the VD for the average chondrule composition is just the halfway of the total evaporation process.

$\mathrm{CI}^{*}$ (the fifth component) represents the average silicate composition of primordial CI-like dust that has to be mixed with the fourth component to form the matrix material after chondrule formation. $\mathrm{CI}^{*}$ is assumed to be omnipresent in PPD, but is distinguished from $\mathrm{CI}^{\text {sil }}$ which is an immediate source of dustons. We take $f_{s}=0.5$ temporarily for $\mathrm{CI}^{*}$. In the following investigation, we have found that Eq. 1 have solutions as long as $\mathrm{CI}^{*}$ with $f_{s}=$ $0.3-1.0$, but not for $\mathrm{CI}^{*}$ with $f_{s}<0.3$ for ordinary chondrites. The $f_{s}$ value of $\mathrm{CI}^{*}$ has an effect on the relative proportion of the matrix to chondrules, but otherwise it does not affect the calculation result. Hence it is taken as a constant.

$A_{m, n}(m=1-4 ; n=1-4)$ are obtained by calculating the evaporative chemical fractionation similar to the one described in "Model calculation of chondrule chemistry" of paper (I). $A_{m, 4}(m=1-4)$ is the chemical composition of the final integrated vapor described above and $A_{m, 5}(m=$ 1-4) is that of $\mathrm{CI}^{*}$.

Calculation proceeds with a step size $q=1.001$ starting with $\mathrm{CI}^{\text {sil }}$ for a specified $f_{s}$, and obtains a set of $A_{m, n}$ at every step number, as described in Paper (I). The $q$ is defined as $X_{A l, k} / X_{A l, k-1}$, where $X_{A l}$ is a molar fraction of $\mathrm{AlO}_{1.5}$ in the MLB and $k$ is an evaporation step number, as described in Paper (I). Equation 1 are solved at every step number to give the molar fractions $F_{n}$. Computation is repeated with $f_{s}$ and step number (viz., VD\%) as parameters so as to satisfy the requirement: $F_{n}(n=1-5)$ to be all positive. Only with this requirement, however, multiple solutions exist. We add another constraint to the calculation, the average composition of chondrules. A unique solution is looked for with the parameters $f_{s}$ and $\mathrm{VD}$, that should reproduce the molar fractions of $\mathrm{Na}$ and $\mathrm{Fe}$ in the average chondrule composition. The choice of Fe is obvious since it is related to the parameter $f_{s}$. The $\mathrm{Na}$ concentration has the largest dynamic range and monotonously changes with VD\% over the multiple solutions, making it easy to find a single solution to fit the exact chondrule average value.

The final solution is the one that should satisfy the bulk composition of chondrite as well as the average composition of chondrules included in that chondrite. The number of constraints for the chondrule is two, while four (viz., $\mathrm{Na}, \mathrm{Mg}, \mathrm{Al}$ and $\mathrm{Si}$ concentrations) for the bulk chondrite. As explained in paper (I), the four constraints is good enough to fit the composition. However, the two constraints are not enough. For this reason a best fitting is not expected for the average chondrule composition. As it turned out, however, the fitting to the chondrule is also quite satisfactory for the two types of chondrites, $\mathrm{L}$ and $\mathrm{H}$.

\subsection{Handling of CAls}

Unlike fitting individual chondrules in paper (I), the bulk chondrite fitting has two sources of complexity, which are nonetheless very important to understand the chemical fractionations in chondrites. The first one is the existence of inclusions in the bulk chondrites: CAIs (Ca-, $\mathrm{Al}$-rich inclusions) and amoeboid olivine aggregates. They are generally thought to be of foreign origin, from their oxygen isotope composition (e.g., Clayton and Mayeda 1977; Hiyagon and Hashimoto 1999) and other isotopic anomalies (e.g., Wasserburg et al. 1977; Wasserburg et al. 2012), having invaded into the chondrite formation zone. CAIs are very enriched in refractory elements and affect the $\mathrm{Al}, \mathrm{Ca}$ and $\mathrm{Ti}$ concentrations in the bulk chondrite composition (e.g., MacPherson 2014, references therein). Amoeboid aggregates are mostly made of forsterite with some refractory elements (e.g., Grossman and Steele 1976; Hashimoto and Grossman 1987). Their compositions are not so different from the bulk chondrite composition and thus hard to be distinguished from the latter in the calculation. For this reason, they are not considered in the calculation.

We identify two types of CAIs: the ones that later invaded into the chondritic materials and the ones which invaded before the chondrule formation. The former are recognized as CAIs as they are clearly visible in certain types of chondrites (mostly carbonaceous chondrites), while the latter are invisible but only manifest their presence as unusual enrichment in refractory elements in chondrules in certain types of chondrites (e.g., $\mathrm{CO}$ chondrites; see Table 1). We call the latter 'ghost CAIs' as we found their existence for the first time.

For the sake of fitting the bulk composition of chondrite that is assumed to consist only of chondrules and matrix, we need to subtract the apparent effect of CAIs that would erroneously raise the concentrations in $\mathrm{Al}, \mathrm{Ca}$ and $\mathrm{Ti}$ in the bulk composition. Therefore, a due amount of CAI is presupposed and subtracted from the bulk composition beforehand. The corrected composition is renormalized to $100 \mathrm{~mol} \%$ (only the silicate with the ten elements) and then applied with the aforementioned calculation procedure. We try typical CAI compositions and then some atypical composition until a best fit is found. The fitted result should give both the CAI-free bulk chondrite composition and the composition of $\mathrm{CAI}$ and its amount, and 
Table 1 Bulk (silicate) chemical compositions of chondrites and average chemical compositions of chondrules (mol\%)

\begin{tabular}{|c|c|c|c|c|c|c|c|c|c|c|c|}
\hline \multicolumn{12}{|c|}{ Chondrite } \\
\hline Type & $\mathrm{NaO}_{0.5}$ & $\mathrm{MgO}$ & $\mathrm{AlO}_{1.5}$ & $\mathrm{SiO}_{2}$ & $\mathrm{KO}_{0.5}$ & $\mathrm{CaO}$ & $\mathrm{TiO}_{2}$ & $\mathrm{CrO}_{1.5}$ & MnO & $\mathrm{FeO}$ & Total \\
\hline \multicolumn{12}{|c|}{ Bulk chondrite } \\
\hline L & 1.917 & 38.366 & 2.760 & 41.335 & 0.146 & 2.063 & 0.094 & 0.436 & 0.300 & 12.584 & 100 \\
\hline $\mathrm{H}$ & 1.921 & 39.938 & 2.905 & 42.155 & 0.132 & 2.147 & 0.104 & 0.474 & 0.302 & 9.921 & 100 \\
\hline LL & 1.838 & 37.512 & 2.634 & 40.508 & 0.127 & 2.053 & 0.098 & 0.426 & 0.296 & 14.510 & 100 \\
\hline$C V$ & 0.518 & 39.356 & 4.243 & 36.656 & 0.041 & 3.045 & 0.137 & 0.465 & 0.172 & 15.368 & 100 \\
\hline E & 1.956 & 39.890 & 3.811 & 49.636 & 0.119 & 1.595 & 0.098 & 0.387 & 0.259 & 2.248 & 100 \\
\hline $\mathrm{CO}$ & 1.369 & 36.873 & 3.629 & 35.612 & 0.084 & 2.431 & 0.105 & 0.391 & 0.221 & 19.285 & 100 \\
\hline $\mathrm{CM}$ & 1.422 & 35.450 & 3.188 & 35.180 & 0.082 & 2.383 & 0.112 & 0.403 & 0.244 & 21.536 & 100 \\
\hline $\mathrm{Cl}$ & 1.835 & 33.208 & 2.653 & 31.931 & 0.114 & 1.906 & 0.077 & 0.419 & 0.293 & 27.563 & 100 \\
\hline $\mathrm{Cl}{ }^{*}$ & 2.128 & 38.517 & 3.077 & 37.035 & 0.133 & 2.210 & 0.090 & 0.486 & 0.340 & 15.984 & 100 \\
\hline \multicolumn{12}{|c|}{ Chondrule } \\
\hline L & 1.765 & 39.358 & 2.631 & 42.223 & 0.134 & 1.864 & 0.089 & 0.456 & 0.284 & 11.197 & 100 \\
\hline $\mathrm{H}$ & 1.913 & 39.393 & 2.735 & 43.740 & 0.147 & 2.023 & 0.104 & 0.435 & 0.301 & 9.208 & 100 \\
\hline $\mathrm{LL}$ & 1.763 & 41.238 & 2.415 & 42.859 & 0.175 & 1.654 & 0.097 & 0.627 & 0.295 & 8.878 & 100 \\
\hline$C V$ & 0.485 & 51.884 & 2.814 & 40.330 & 0.041 & 2.782 & 0.145 & 0.228 & 0.058 & 1.233 & 100 \\
\hline E & 1.237 & 43.853 & 1.939 & 48.802 & 0.129 & 1.373 & 0.046 & 0.257 & 0.155 & 2.208 & 100 \\
\hline $\mathrm{CO}$ & 1.386 & 41.717 & 4.240 & 40.456 & 0.047 & 3.689 & 0.145 & 0.440 & 0.167 & 7.712 & 100 \\
\hline CM & 0.039 & 52.775 & 1.422 & 41.518 & 0.000 & 2.161 & 0.203 & 0.328 & 0.087 & 1.467 & 100 \\
\hline
\end{tabular}

also the whole rock composition including CAIs, which should be identical to the bulk chondrite composition on the ten element basis.

Ghost CAIs are handled in the opposite way. Extra Al, $\mathrm{Ca}$ and $\mathrm{Ti}$ (and also $\mathrm{Mg}$ and $\mathrm{Si}$ if necessary) in due amounts are added to the initial composition $\mathrm{CI}^{\text {sil }}$, and renormalized to $100 \mathrm{~mol} \%$ to give a corrected initial composition for the calculation. This corrected initial composition is applied to the aforementioned calculation procedure in order to fit the bulk chondrite composition. We try the simplest Al:Ca:Ti proportion for the added CAIs, which is identical to the CI-chondritic (or solar abundance) ratio and then some atypical proportion until a best fit is found. The fitted result should give the composition of ghost CAI and its amount and also the whole rock composition which should be identical with the bulk chondrite composition.

As will be shown later, both CAIs and ghost CAIs are identified in two types of chondrites (CO and LL). To these chondrites the combination of the two procedures described above is applied.

\subsection{Correction for the Effect of Aqueous Alteration in the Bulk Chondrites}

Another source of complexity is the aqueous alteration of bulk chondrites suggested for certain types of chondrites (e.g., Tomeoka and Buseck 1985; Krot et al. 1998), that should have occurred after accumulation of chondritic components onto a large body, or meteorite parent body as usually supposed. This would have changed the redox state of Fe-bearing phases towards more oxidizing. Specifically Fe metals would have been changed into Fe oxides that could be accommodated into silicate or exist as a certain hydrous oxide phase. The oxidation should be far more effective for matrix materials because of their finegrained and porous nature than for chondrules which are coarse-grained and non-porous. This effect should be removed for the present calculation to be properly executed because it is a secondary effect that has nothing to do with the chondrule formation. The problem is how to recognize and distinguish it from the original redox state of chondrite forming materials before alteration.

A hint is the difference in redox state between the chondrules and the matrix (or the bulk chondrite). The wet-chemical analyses of chondrites distinguish Fe oxides from metals and sulfides. Therefore, the $\mathrm{FeO}$ content in the bulk chondrite is known. If it is too abundant as compared with the average $\mathrm{FeO}$ content in the chondrules, we should suspect the effect of alteration in the matrix. In order to compensate the $\mathrm{FeO}$ content in the bulk chondrite for the effect of alteration, we introduce a 
factor $f_{w}$ which is the ratio of the $\mathrm{FeO}$ concentration in the alteration-free bulk chondrite relative to that in the wet-chemical data.

The $f_{w}$ is a factor which is imposed on the $\mathrm{FeO}$ concentration in the wet-chemical bulk chondrite data, but not on the average composition of chondrules which are contained in that chondrite. We parameterize $f_{w}$ just like $f_{s}$ in the calculation and find a best fitting value. As it turned out, it is rather easy to find the best fit value within relative errors of $10 \%$ or so since other values just do not allow equations to have solutions. As shown later, $f_{w}$ is unity for $\mathrm{E}, \mathrm{H}$ and $\mathrm{L}$ chondrites, while other four types of chondrites have values smaller than unity, implying that aqueous alteration was in action in the latter four chondrites after their accretion to large bodies.

\subsection{Data}

Table 1 shows (in the upper columns) the bulk chemical compositions of seven types of chondrites, as well as two $\mathrm{CI}$ compositions, on the ten oxide element basis, and also shows (in the lower columns) the average compositions of chondrules contained in each type of chondrites, used in the calculation. The data sources and some adjustments to data are explained in Appendix A.

\section{Results}

In the followings we explain the results of numerical fittings in detail for chondrites temporarily grouped according to the fitting procedure commonly applied.

\subsection{Ordinary Chondrites ( $\mathrm{L}, \mathrm{H}$ and LL types)}

Ordinary chondrites are generally presumed CAI-free. Thus the fitting procedure is simple. We obtained a single solution for each chondrite. Figure 1 shows the results of numerical fittings for $\mathrm{L}, \mathrm{H}$ and LL types of chondrites, by normalizing the abundance of the elements to the $\mathrm{CI}$ and $\mathrm{Si}$ abundance. The determined parameters as well as proportions of the five components and compositions thereof are thoroughly examined in Section 3.5.

Here we investigate status of the fitting element by element. The four elements calculated, $\mathrm{Na}, \mathrm{Mg}, \mathrm{Al}$ and $\mathrm{Si}$ fit exactly the bulk chondrite composition simply because their concentrations are given as known values in the five simultaneous linear equations. The Fe also fits nearly perfectly because it is controlled with parameter $f_{s}$. The remaining five elements, $\mathrm{K}, \mathrm{Ca}, \mathrm{Ti}, \mathrm{Cr}$ and $\mathrm{Mn}$, are left free of preset constraints. As shown in Fig. 1 ('calc bulk' on the left panels), the calculated concentrations in these elements are more or less different from the bulk chondrite values, except for $\mathrm{Ca}$ in $\mathrm{L}$ and $\mathrm{H}$ chondrites. There must be reason or cause for the elements that are not fitted well, which will be explored below.
There are at least two kinds of causes possible. The first is the possibility that the bulk composition and/or the average chondrule composition adopted for the calculation is in error. As long as the bulk compositions of ordinary chondrites are concerned, this possibility is small because only the 'falls' meteorites are used and the average of many chondrite wet-chemical analyses (see Appendix A) are used in the calculation. On the other hand, the EPMA analyses of chondrule compositions may have their own source of error, especially the broad-beam corrections as described in paper (I). Minor elements, i.e., $\mathrm{K}, \mathrm{Ti}, \mathrm{Cr}$ and $\mathrm{Mn}$, may suffer from the problem of detection limits in the EPMA analysis.

Another possibility may be related with the validity including our original experiments that were translated to the volatilities of elements used in the present calculation and our model assumptions about the chondrule and chondrite formation, or with some essential problem such as the initial composition assumed for the primordial dust and some possible effect not included in the calculation.

A survey of the bulk chondrite fitting in Fig. 1 indicates the same trends in all three ordinary chondrites that 1 ) K, $\mathrm{Ti}$ (and maybe $\mathrm{Ca}$ ) are depleted in the calculation relative to the actual chondrites and 2) $\mathrm{Cr}$ and $\mathrm{Mn}$ in the calculation are too enriched. The same trends are also seen in the average chondrule fitting in Fig. 1 ('calc cdl ave' on the right panels). The fact implies that these trends are not related with analytical errors in the chondrite and chondrule analyses. They might be attributed to some problem in our calculation, although we cannot think of any defect at the moment. Let us try to find a positive reason for the observed trends. They could originate in the chondrule formation process or be ascribed to the primary features of the dust.

The depletion in $\mathrm{Cr}$ and $\mathrm{Mn}$ in the actual chondrites and chondrules relative to the calculation may be due to their having partially existed as sulfides in the primordial dust just like Fe-sulfide, which would decrease their concentrations in the silicate dust. $\mathrm{Mn}$ and $\mathrm{Cr}$ are multi-valent elements like $\mathrm{Fe}$, although they are usually considered most stable as oxides (viz., silicate) in PPD. Mn-bearing and Crbearing iron sulfides are often observed in a reduced type of chondrites such as $\mathrm{E}$ chondrites. Some $\mathrm{Cr}$ can dissolve into $\mathrm{Fe}-\mathrm{Ni}$ alloy. For fitting the $\mathrm{Cr}$ and $\mathrm{Mn}$ concentrations in the bulk ordinary chondrites, $\sim 20 \%$ of $\mathrm{Cr}$ and $\sim 25 \%$ of Mn must be removed from the initial $\mathrm{CI}^{\text {sil }}$ composition. There are two possibilities. (1) $\mathrm{Mn}$ and $\mathrm{Cr}$ are partially removed in the form of metal and/or sulfide by the metal (+sulfide) - silicate fractionation process, as proposed for explaining the Fe depletion in bulk chondrites relative to CI (Urey and Craig 1953; Larimer and Anders 1970). (2) The wet chemical analyses of bulk chondrites by Jarosewich (1990) do not include the Mn and Cr contents 
Table 2 Depletion factors for $\mathrm{Cr}$ and $\mathrm{Mn}$

\begin{tabular}{llllllll}
\hline \multicolumn{7}{c}{ Chondrite Type } \\
& CV & CM & E & CO & LL & H & L \\
\hline $\mathrm{Cr}$ & 1.17 & 1 & 0.55 & 0.95 & 0.8 & 0.83 & 0.78 \\
$\mathrm{Mn}$ & 0.5 & 0.75 & 0.46 & 0.65 & 0.72 & 0.73 & 0.74 \\
\hline
\end{tabular}

contributed by sulfide and metal. The both possibilities are likely combined to make $\mathrm{Mn}$ and $\mathrm{Cr}$ depleted in the bulk chondrite compositions relative to the calculation.

Table 2 shows the depletion factors to be imposed on the initial $\mathrm{CI}^{\text {sil }}$ for $\mathrm{Cr}$ and $\mathrm{Mn}$ to fit the bulk chondrite compositions for seven types of chondrites investigated here. E chondrite, the most reduced type, requires nearly halves of $\mathrm{Cr}$ and $\mathrm{Mn}$ to be removed from the initial silicate composition. On the other hand, CV and CM (and possibly $\mathrm{CO}$ ) requires no depletion in $\mathrm{Cr}$, contrary to $\mathrm{Mn}$. This might suggest that $\mathrm{Cr}$ favors oxide more than Mn does.

The consistent depletion in $\mathrm{K}$ in the calculation (Fig. 1) as compared to the bulk composition and the average chondrule composition is mysterious, especially knowing the fact that the fitting of $\mathrm{K}$ is nearly perfect for the other four types of chondrites as will be shown later. Reexamination on the relative volatilities indicates that a factor of two or three more reduction in the relative volatility of $\mathrm{K}$ compared to $\mathrm{Na}$ is required to explain the $\mathrm{K}$ enrichment in the bulk ordinary chondrites. Such an adjustment is against our experimental results which indicated similar relative volatilities for $\mathrm{Na}$ and $\mathrm{K}$ within errors of a few tens

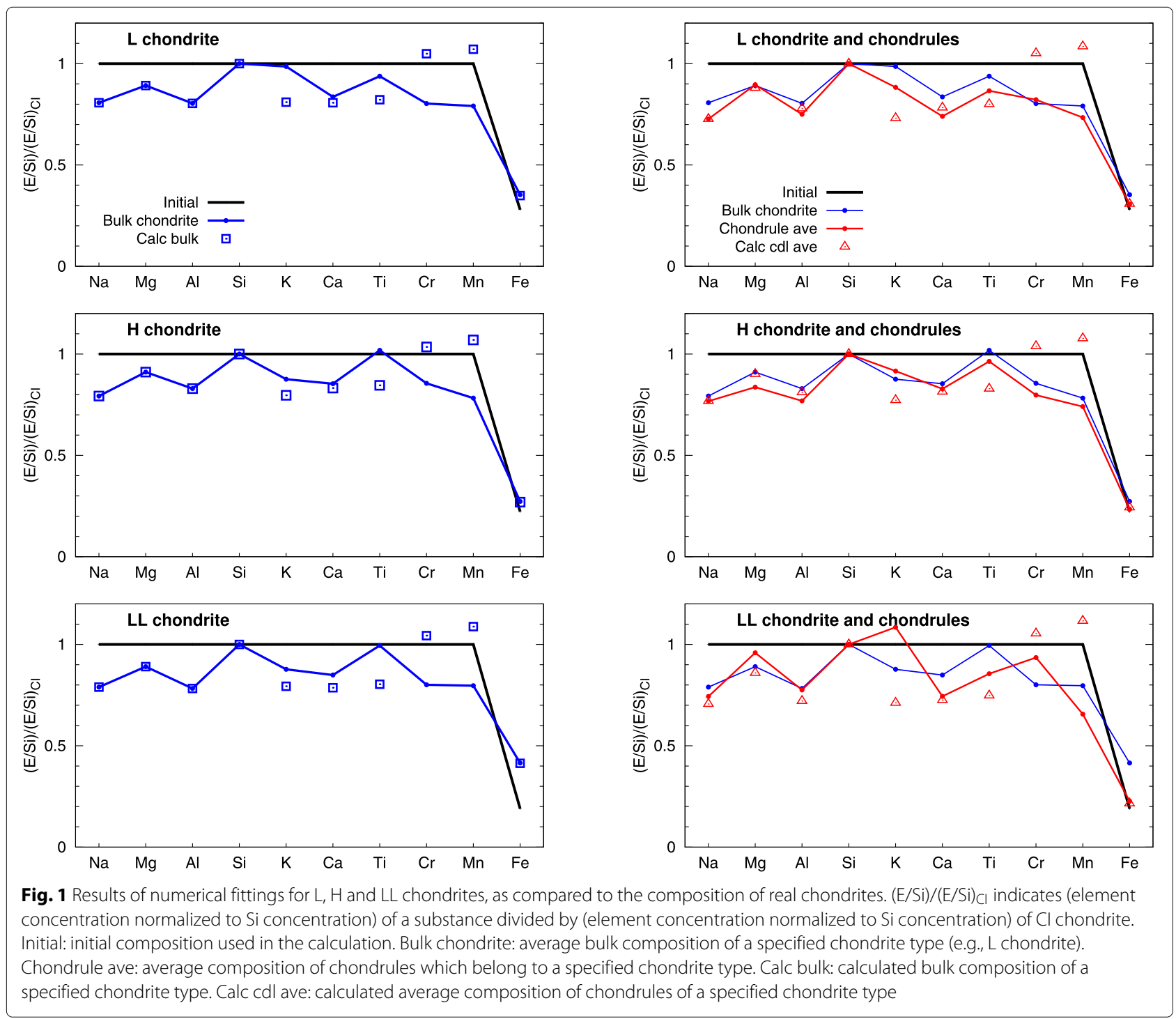


of percent (Nakano and Hashimoto 2020). If the relative volatility of $\mathrm{K}$ was adjusted to fit the ordinary chondrites, it should fail for the other four chondrite types.

We observe a consistent depletion in Ti (and possibly in $\mathrm{Ca}$ ) in the calculation relative to both the bulk composition and the average chondrule composition of ordinary chondrites. This implies some enrichment in these elements before the chondrule forming process. It should be related to a CAI invasion before the formation of chondrules, although ordinary chondrites are generally thought being free of CAIs. We will re-examine ordinary chondrites later.

\subsection{Carbonaceous Chondrites and Enstatite Chondrites}

Carbonaceous chondrites contain CAIs up to several volume percent, and need subtraction of their effects before fitting their bulk compositions and average chondrule compositions. Fitting is a process of trial and error by assuming the composition and amounts of CAIs to subtract from the wet-chemical composition of bulk silicate chondrite, while surveying the other parameters, VD, $f_{s}$ and $f_{w}$ at the same time. Let such a tentative, CAI-free bulk composition to be fitted be called, a 'CAI-subtracted bulk composition'. We apply the regular calculation procedure to the CAI-subtracted bulk composition and the average chondrule composition. When the best fitting parameters and appropriate CAI component are found, the bulk composition of chondrite is reconstructed by adding the CAI component, called 'the calculated bulk composition'.

Chondrules in $\mathrm{CO}$ chondrites are unusually rich in $\mathrm{Al}, \mathrm{Ca}$ and $\mathrm{Ti}$ as compared to chondrules in any other types of chondrites, as shown in Table 1. The abundances of these elements are 1.6-1.9 times as high as those in CI. This fact suggests that the initial composition of the primordial dust had been pre-enriched in these refractory elements, most probably in the form of invaded CAIs, before the chondrule formation of $\mathrm{CO}$ chondrites. We call those CAIs 'ghost CAIs' because they should have become invisible by mixing with the primordial dust during high-temperature melting in the chondrule forming process, but would only appear in the chemistry of the chondrules thus formed. Therefore, CAIs with an adequate composition in an adequate amount must be added to the initial composition $\mathrm{CI}^{\text {sil }}$ before fitting the bulk and chondrule composition of $\mathrm{CO}$ chondrites. We call such a mixed composition a 'CAI-loaded CI silicate composition'. CO chondrites also contain visible CAIs, which can be handled with subtraction of due amounts of CAI just as other carbonaceous chondrites.

Enstatite chondrites are rich in $\mathrm{Al}$ in their bulk composition, next to $\mathrm{CV}$ as shown in Table 1, although they do not belong to carbonaceous chondrites. As a matter of fact, no solution is obtained without subtracting (Al-rich) CAI before fitting. We have found that $2.3 \mathrm{~mol} \%$ of an $\mathrm{Al}-$ rich CAI $\left(\mathrm{Al}_{0.79} \mathrm{Ca}_{0.19} \mathrm{Ti}_{0.02}\right.$; a mixture of more than two phases is possible) is needed to mix after the chondrule formation to reproduce the bulk and chondrule composition of $\mathrm{E}$ chondrites. The $\mathrm{Ca} / \mathrm{Al}$ ratio of $\mathrm{E}$ chondrites far lower than that of $\mathrm{CI}$ has been an enigma for long. It has been suggested that $\mathrm{Ca}$ was mobilized by shock and localized somewhere in the chondrite (Rubin et al. 2009). However, no such occurrence has been found yet. We suggest that $\mathrm{E}$ chondrite is unusually enriched in $\mathrm{Al}$ due to the incorporation of $\mathrm{Al}$-rich $\mathrm{CAI}$ after the chondrule formation.

Figure 2 shows the results of numerical fittings for $\mathrm{CV}, \mathrm{CM}, \mathrm{CO}$ and $\mathrm{E}$ chondrite. In all of these figures, the bulk $\mathrm{Cr}$ and $\mathrm{Mn}$ abundances are already fitted with the depletion factors shown in Table 2. The bulk Al, Ca and $\mathrm{Ti}$ abundances are fitted with the CAI composition and its amount of subtraction or addition as described above. $\mathrm{CV}, \mathrm{CM}$ and $\mathrm{E}$ chondrites require mixing of CAIs after the chondrule formation. $\mathrm{CO}$ chondrites require preenrichment in $\mathrm{Al}, \mathrm{Ca}$ and $\mathrm{Ti}$ (as ghost CAIs) twice as abundant as $\mathrm{CI}$ before the chondrule formation, while only a few percent addition of CAIs to the bulk chondrite afterwards. $\mathrm{K}$ is left with no fitting effort, which nevertheless shows an extraordinary match between the calculation and the bulk chondrite for all four types of chondrites, as seen in Fig. 2. This is in contrast with the result for ordinary chondrites, which clearly shows a disagreement between the calculation and the bulk chondrites.

The CAIs in CV, CM and $\mathrm{CO}$ chondrites predicted by the calculation have chemical compositions of rather common CAIs with the Al:Ca:Ti ratio similar to that of $\mathrm{CI}$ composition, consistent with the observation in the real chondrites. The ghost CAIs predicted for $\mathrm{CO}$ chondrites also have the same Al:Ca:Ti ratio as CI. The CAIs predicted in $\mathrm{E}$ chondrites, however, are very rich in $\mathrm{Al}$ and most probably poor in $\mathrm{Mg}$ and $\mathrm{Si}$. Such a CAI exists in carbonaceous chondrites, though extremely small in concentration (e.g., MacPherson et al. 1983; Ireland 1988), as compared with common CAIs. In this sense $\mathrm{E}$ chondrites, having only Al-rich CAIs predicted, are quite unique and require $\mu \mathrm{m}$-scaled petrographic study since Al-rich CAIs are usually very small in size. Actually Bischoff et al. (1984) and Guan et al. (2000) have reported such occurrences.

The average chondrule compositions are not so well reproduced by the calculation compared to the bulk compositions, particularly for $\mathrm{CV}$ and $\mathrm{CM}$ chondrites. It is very likely that the number of chondrules used for averaging is too small, only eight for $\mathrm{CV}$ and ten for $\mathrm{CM}$, to be representative of chondrules in those chondrites. Nevertheless the matching to the average chondrule is quite good for $\mathrm{Mg}$ and $\mathrm{Si}$, reconfirming the validity of our study. 


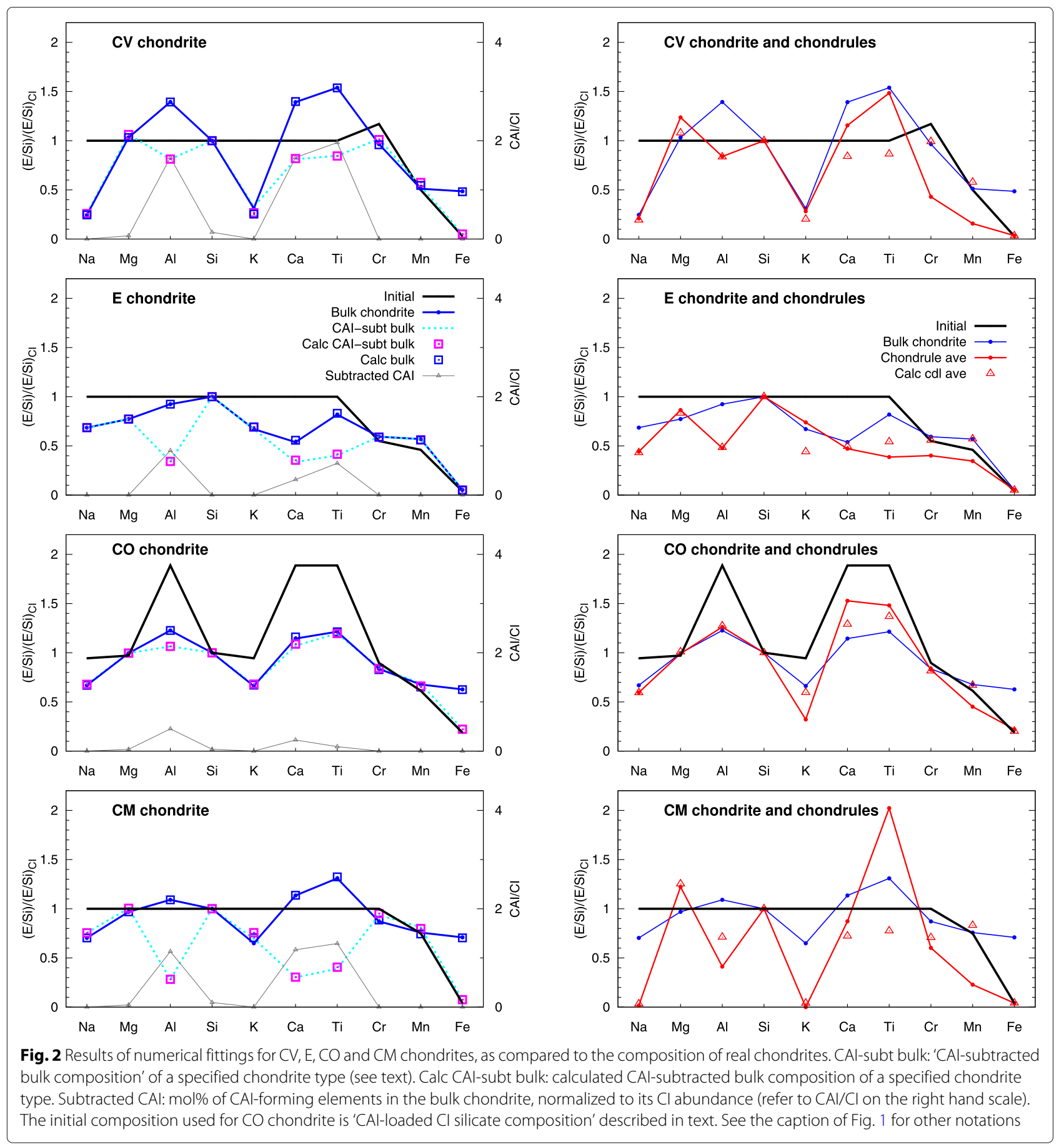

\subsection{Ordinary Chondrites Revisited}

Knowing a possible existence of ghost CAIs before the chondrule formation in $\mathrm{CO}$ chondrites, we hypothesize a pre-mixing of Ti-rich CAI into the primordial dust before chondrule formation, to explain the depletion in $\mathrm{Ti}$ and possibly in $\mathrm{Ca}$ in the calculation for ordinary chondrites.
Figure 3 shows the calculated bulk compositions as well as the calculated average chondrule compositions for the three types of ordinary chondrites, along with the real chondrite compositions for comparison. As in Fig. 2, the bulk $\mathrm{Cr}$ and $\mathrm{Mn}$ abundances are fitted with the depletion factors shown in Table 2. The CAI-loaded CI silicate composition explained in Section 3.2, shown in bold black 


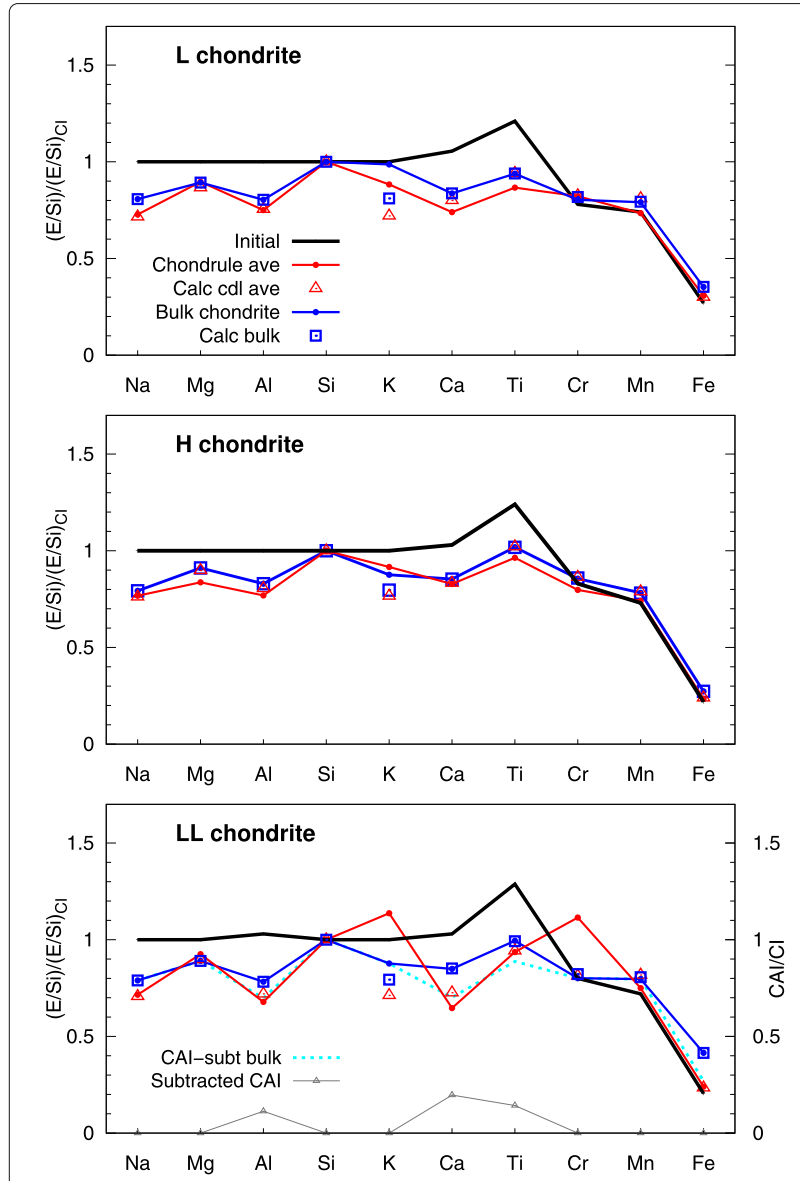

Fig. 3 Results of numerical fittings for $L, H$ and $L L$ chondrites, that incorporate the effect of CAls and ghost CAls. See the captions of Figs. 1 and 2 for notations lines in the figures, includes the contribution of ghost CAIs to the $\mathrm{CI}^{\text {sil }}$. In case of LL chondrites, the CAI composition to be added after chondrule formation is also shown in thin gray line. The final matching is quite satisfactory for the bulk compositions with the $\mathrm{K}$ problem remaining persistently, and is fairly good for the average chondrule compositions.

Table 3 shows the amount of oxide elements to be entered as ghost CAIs to the primordial CI-like silicate,

Table 3 Oxide elements entered as Ghost CAls to $\mathrm{Cl}^{\text {sil }}$ before chondrule fromation (mol\%)

\begin{tabular}{clllll}
\hline Chondrite & $\mathrm{AlO}_{1.5}$ & $\mathrm{CaO}$ & $\mathrm{TiO}_{\mathbf{2}}$ & $\mathbf{M g O}$ & $\mathbf{S i O}_{\mathbf{2}}$ \\
\hline $\mathrm{CO}$ & 3.109 & 2.234 & 0.091 & 1.117 & 2.234 \\
$\mathrm{LL}$ & 0.102 & 0.073 & 0.028 & - & - \\
$\mathrm{H}$ & - & 0.073 & 0.024 & - & - \\
$\mathrm{L}$ & - & 0.131 & 0.020 & - & - \\
\hline
\end{tabular}

calculated for the four types of chondrites. CO chondrites require ghost CAIs as much as $9 \mathrm{~mol} \%$ in their initial silicate composition. The best fitting was made with a common CAI composition, viz., having the Al:Ca:Ti ratio being identical to that of $\mathrm{CI}$ composition and the $\mathrm{Mg}$ and $\mathrm{Si}$ in half or equal amount of $\mathrm{Ca}$, respectively. LL, $\mathrm{H}$ and $\mathrm{L}$ chondrites, however, require Ti-enriched, Al-Ca-Ti oxides for their ghost CAIs. The required mass for the ghost CAIs, relative to the $\mathrm{CI}^{\text {sil }}$, before chondrule formation is only $0.1,0.15$ and $0.2 \mathrm{~mol} \%$ for $\mathrm{H}, \mathrm{L}$ and $\mathrm{LL}$, respectively.

In Section 3.2 we suggested invasion of the Al-rich CAI to $\mathrm{E}$ chondrites after chondrule formation. Its mass, 2.3 mol\%, however, is far larger than those for ghost CAIs for ordinary chondrites, and its composition is not $\mathrm{Ti}^{-}$ enriched but $\mathrm{Ca}$-depleted relative to the $\mathrm{CI}$ composition. Thus the ghost CAIs for ordinary chondrites are not only different from that for $\mathrm{CO}$ chondrites but from the Al-rich $\mathrm{CAI}$ in $\mathrm{E}$ chondrites.

\subsection{Question of $\mathrm{Cl}$ versus Solar Photosphere}

So far we have assumed that the initial composition of the primordial silicate dust was the CI composition throughout the present work, with the FeO content controlled with the parameter $f_{s}$. Figure 4 shows the elemental abundances in bulk CI chondrites and those in the solar photosphere, in various compilations by Lodders (2003); Lodders et al. (2009); Palme et al. (2014), normalized to the CI chondritic abundances and the element Si of Lodders (2003), viz., those used in the present work. They more or less agree with each other, with exceptions of $\mathrm{Ti}$ and $\mathrm{Mn}$.

The most important in the chemical fractionation is the $\mathrm{Mg} / \mathrm{Si}$ ratio since they are the most abundant elements in the silicate. The solar photospheric elemental abundance (SP) in Lodders (2003) has $\mathrm{Mg} / \mathrm{Si}=1.00$ as compared to $\mathrm{Mg} / \mathrm{Si}=1.04 \mathrm{in} \mathrm{CI}$ in Lodders (2003). The difference of $4 \%$

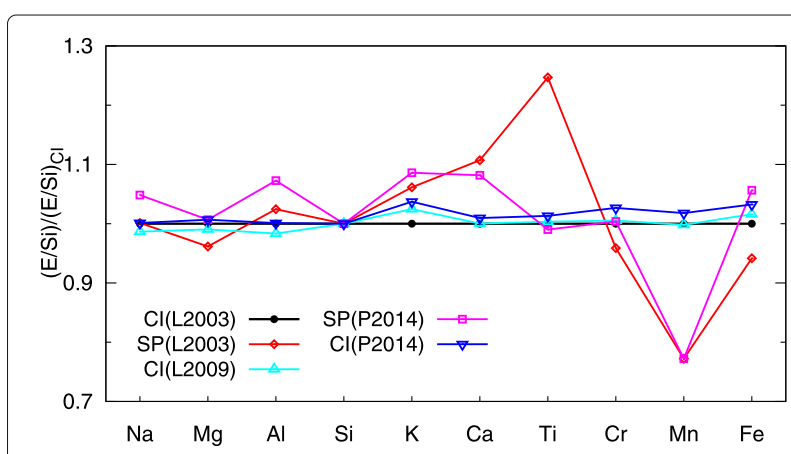

Fig. 4 The elemental abundances from various sources, normalized to the Cl elemental abundances of Lodders (2003). Cl: Cl chondrite. SP: solar photosphere. L2003: Lodders (2003). L2009: Lodders et al. (2009). P2014: Palme et al. (2014) 
has only a small effect on the fitted parameters such as VD and $f_{s}$, as we performed calculations using both SP and CI abundances.

The $\mathrm{Ti}$ abundance is questioned. Is the $\mathrm{Ti}$ in $\mathrm{CI}$ anomalously low? Or is the Ti in SP in Lodders (2003) anomalously high? The $\mathrm{CI}$ composition is the average of the three $\mathrm{CI}$ chondrites. Its $\mathrm{Ti}$ concentration has a very small dispersion relative to its average value, $\delta \mathrm{Ti} / \mathrm{Ti} \sim 0.02$, much smaller than the normal analytical error in $\mathrm{Ti}$ analysis. Therefore the $\mathrm{Ti}$ abundance in $\mathrm{CI}$ is trusted. If, on the other hand, the Ti in SP (Lodders 2003) is used in the present calculation, invasion of the Ti-enriched, $\mathrm{Al}-\mathrm{Ca}-\mathrm{Ti}$ oxides is not needed to fit the bulk compositions of ordinary chondrites, because of the high abundance of $\mathrm{Ti}$ in $\mathrm{SP}$ relative to $\mathrm{CI}$ as seen in Fig. 4. In a new compilation of SP abundances by Palme et al. (2014), the Ti abundance is revised to the value nearly identical to that of the CI in Lodders (2003). Then the problem of the high Ti abundance for ordinary chondrites persists, and should be explained by the invasion of Ti-enriched, Al-Ca-Ti oxides as ghost CAIs as described in Section 3.3.

The Mn abundance in SP (Palme et al. 2014) has not been revised. Hence $\mathrm{Mn}$ in SP is still $\sim 25 \%$ lower than its $\mathrm{CI}$ abundance. If the SP abundance was used for $\mathrm{Mn}$, the depletion factors imposed on it for ordinary chondrites, $0.72-0.74$ would become $\sim 1$, requiring no depletion for $\mathrm{Mn}$. For those chondrites with larger depletion factors such as $\mathrm{CV}, \mathrm{E}$ and $\mathrm{CO}$, however, the depletion problem would remain.

In the revised SP (Palme et al. 2014), $\mathrm{K}$ is enriched by $\sim 10 \%$ compared to the CI (Lodders 2003). It would compensate the deficit in $\mathrm{K}$ in ordinary chondrite types at least in $\mathrm{H}$ and LL. On the other hand, it would cause enrichment in $\mathrm{K}$ for the other four chondrites as they are perfectly matched with the CI value, as described in Section 3.2.

The analytical precision in determining concentrations of elements are far better in the laboratory chemical analyses than those in the stellar spectrophotometry. Especially the abundances of minor and trace elements recommended for the solar system abundances depend on the meteorite (CI) analyses. On the other hand, the solar photosphere is no doubt representative of the solar system elemental abundances. We rely on CI simply because its elemental abundance is very similar to that of the solar photosphere.

\subsection{Comparison between Chondrites}

We infer probable reasons from the fitted parameters and the determined unknowns, why the chemical variations among the chondrites resulted from the same source material, viz., CI-like primordial dust.

Figure 5 (upper panel) and Table 4 show the VD\% for various chondrite types. Here the obtained VD indicates

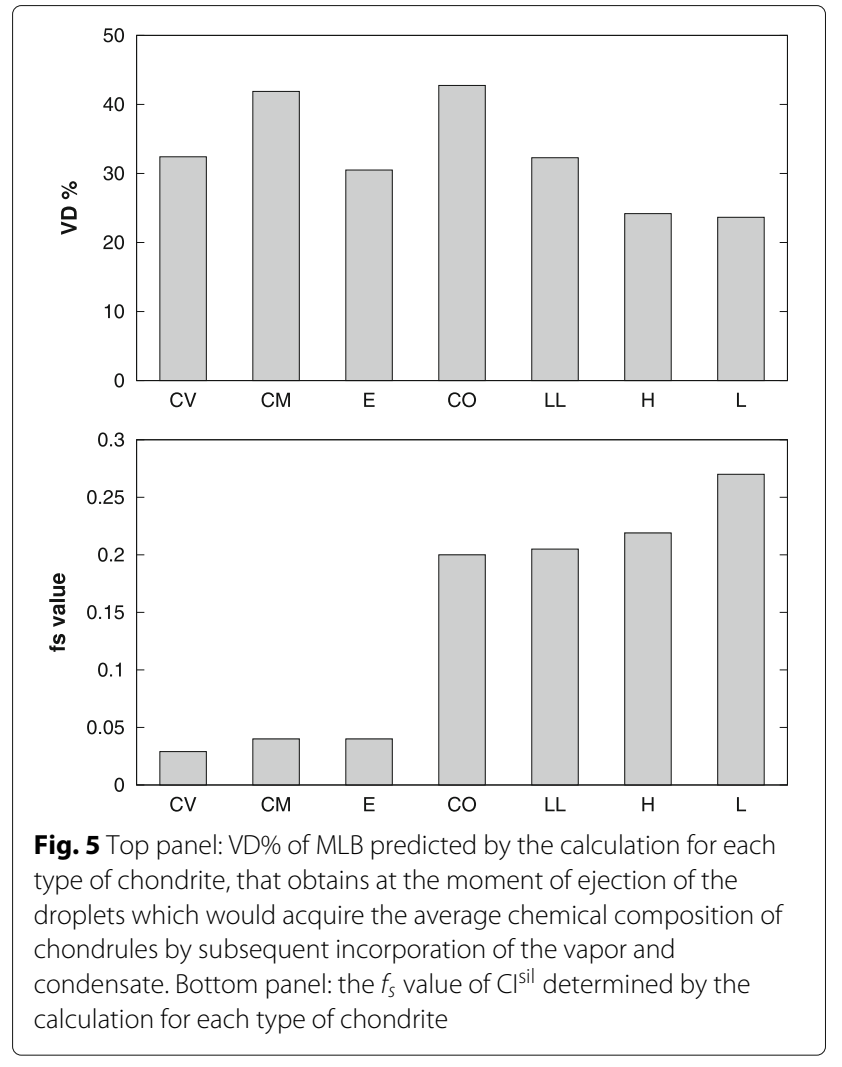

the vaporization degree of MLB at the moment when the chondrules with the average chondrule composition are produced as jet-droplets from the MLB. Chondrules have a wide range of $\mathrm{VD}$ value as already analyzed in paper (I). Here the VD for a specified chondrite type is the one that satisfies both its bulk composition and the average composition of chondrules included in it. The VD is $\sim 40 \%$ for $\mathrm{CM}$ and $\mathrm{CO}$ chondrites, $\sim 30 \%$ for $\mathrm{CV}, \mathrm{E}$ and $\mathrm{LL}$ and $\sim 25 \%$ for $\mathrm{H}$ and $\mathrm{L}$. We have assumed in the model calculation that the VD of the average chondrule is just a half of the final VD reached

Table 4 Results of the calculation

\begin{tabular}{llllllll}
\hline & & & \multicolumn{5}{c}{ Molar proportion; total = 1 } \\
\cline { 6 - 8 } Chd & VD\% & $\boldsymbol{f}_{\boldsymbol{s}}$ & $\boldsymbol{f}_{\boldsymbol{w}}$ & Cdls & Mtx & CAls & Ghost CAls \\
\hline CV & 32 & 0.03 & 0.1 & 0.84 & 0.11 & 0.0552 & 0 \\
CM & 42 & 0.04 & 0.1 & 0.25 & 0.68 & 0.0741 & 0 \\
E & 30 & 0.04 & 1 & 0.62 & 0.35 & 0.0226 & 0 \\
CO & 43 & 0.20 & 0.35 & 0.68 & 0.26 & 0.0156 & 0.0391 \\
LL & 32 & 0.21 & 0.65 & 0.74 & 0.25 & 0.0060 & 0.0009 \\
H & 24 & 0.22 & 1 & 0.80 & 0.20 & 0 & 0.0005 \\
L & 24 & 0.27 & 1 & 0.66 & 0.34 & 0 & 0.0007 \\
\hline
\end{tabular}

Note. Chd: Chondrite. Cdls: Chondrules. Mtx: Matrix 
by MLB. Then the final VD reached would be twice as large as the VD, viz., $50-80 \%$. These values are consistent with the calculation made for individual chondrules in paper (I).

Figure 5 (lower panel) and Table 4 show the $f_{s}$ values for various chondrite types. $\mathrm{CV}, \mathrm{CM}$ and $\mathrm{E}$ chondrites have small $f_{s}$ values, $0.03-0.04$, while $\mathrm{CO}, \mathrm{LL}, \mathrm{H}$ and $\mathrm{L}$ have relatively high $f_{s}$ values, $0.20-0.27$. The definition of $f_{s}$ is the molar fraction of oxidized Fe relative to the total Fe contained in the primordial dust having the CI composition. Therefore, it is likely that the determined $f_{s}$ values reflect the original redox state of the primordial dust before the chondrule formation. $\mathrm{CV}$ and $\mathrm{CM}$ chondrites are usually considered as oxidized types, but their $f_{s}$ values suggest that they were originally reduced.

The $f_{w}$ values in Table 4 showing 0.1 for fitting CV and $\mathrm{CM}$ chondrites suggest that they were aqueously altered and oxidized after accumulation onto their parent bodies. $\mathrm{CO}$ and LL chondrites showing $f_{w}<1$ also suggest their post-accretional aqueous alteration. $\mathrm{E}, \mathrm{H}$ and $\mathrm{L}$ chondrites having $f_{w}=1$, on the other hand, are considered free of alteration.

Figure 6 and Table 5 show molar proportions of the five components for the bulk composition and of the three components for the average chondrule composition, determined by the calculation. We can recognize four patterns in Fig. 6. CV is characterized by its high $F_{1}$ and
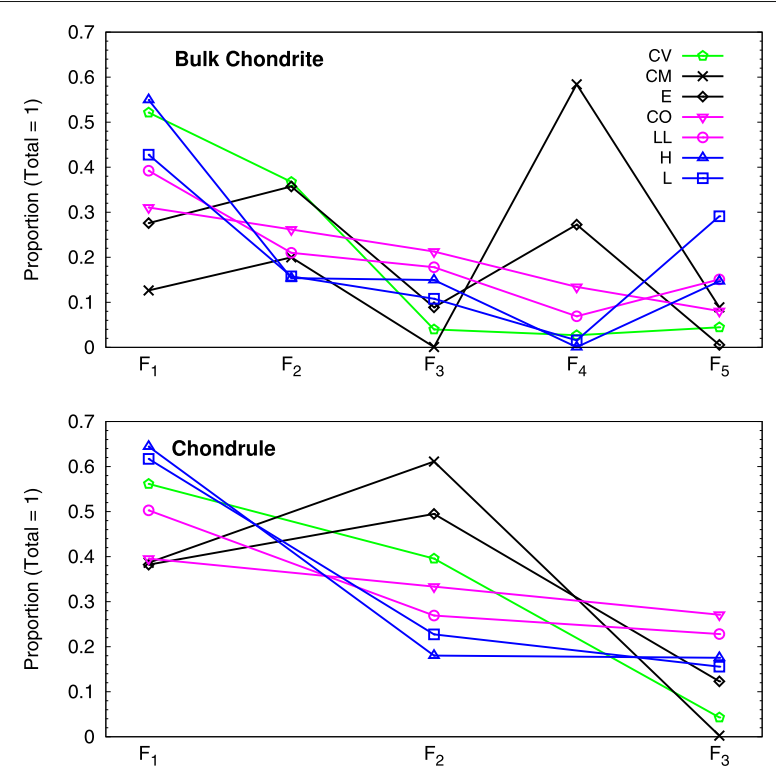

Fig. 6 Top panel: Proportions of the five components, $F_{n}$ 's in the bulk chondrite determined by the calculation for each chondrite type. $F_{1}$ : jet-droplet. $F_{2}$ : differential vapor. $F_{3}$ : integrated vapor. $F_{4}$ : final integrated vapor. $F_{5}: \mathrm{Cl}^{*}$ dust. Bottom panel: Proportions of the three components used for formation of the average chondrule
Table 5 Molar fractions of components for the bulk chondrite and for the chondrules

\begin{tabular}{|c|c|c|c|c|c|c|c|c|}
\hline \multirow{3}{*}{ Chd } & \multicolumn{8}{|c|}{ Molar Fraction of Components } \\
\hline & \multicolumn{5}{|c|}{ Bulk chondrite; total = 1} & \multicolumn{3}{|c|}{ Ave. cdl; total = 1} \\
\hline & $F_{1}$ & $F_{2}$ & $\mathrm{~F}_{3}$ & $\mathrm{~F}_{4}$ & $\mathrm{~F}_{5}$ & $\mathrm{~F}_{1}$ & $F_{2}$ & $\mathrm{~F}_{3}$ \\
\hline CV & 0.521 & 0.367 & 0.040 & 0.027 & 0.045 & 0.562 & 0.396 & 0.043 \\
\hline CM & 0.126 & 0.200 & 0.001 & 0.584 & 0.089 & 0.386 & 0.611 & 0.002 \\
\hline$E$ & 0.276 & 0.357 & 0.089 & 0.273 & 0.005 & 0.382 & 0.495 & 0.123 \\
\hline $\mathrm{CO}$ & 0.311 & 0.262 & 0.213 & 0.134 & 0.081 & 0.396 & 0.333 & 0.271 \\
\hline $\mathrm{LL}$ & 0.392 & 0.210 & 0.178 & 0.069 & 0.151 & 0.503 & 0.269 & 0.228 \\
\hline $\mathrm{H}$ & 0.549 & 0.154 & 0.150 & 0.000 & 0.147 & 0.644 & 0.180 & 0.175 \\
\hline$L$ & 0.428 & 0.158 & 0.108 & 0.015 & 0.291 & 0.617 & 0.227 & 0.156 \\
\hline
\end{tabular}

Note. Chd: chondrite type

Ave. cdl: Chondrules having their average composition

$F_{2}$ proportions as compared to low proportions in $F_{3}, F_{4}$ and $F_{5} . \mathrm{CM}$ is relatively enriched in $F_{2}$ and $F_{4}$. E resembles $\mathrm{CM}$ in its overall pattern. $\mathrm{CO}$ is characterized by its monotonous decrease from $F_{1}$ through $F_{5} . \mathrm{H}$ and $\mathrm{L}$ are enriched in $F_{1}$ and $F_{5}$ while very depleted in $F_{4}$. LL may be recognized between the $\mathrm{CO}$ and the $\mathrm{H}$ and $\mathrm{L}$ patterns.

Figure 7 shows the chemical compositions of the five components for three types of chondrites. The components have their own characteristic compositions, but they are similar between different types of chondrites. Therefore, it is the difference in proportion among the five components that distinguishes and determines the bulk composition and the average chondrule composition of a specific type of chondrite. Specifically, E chondrites are Si-rich for the reason that they contain more $F_{2}$ (differential vapor) and $F_{4}$ (final integrated vapor), which are enriched in $\mathrm{Si}$ but depleted in $\mathrm{Mg}$, as shown in Figs. 6 and 7.

Figure 8 (top panel) shows the molar proportion ${ }^{1}$ of chondrules in the bulk chondrite, determined by the calculation. The rest, (1 - chondrule), includes all other components in the chondrite. The molar proportions of chondrule, matrix, CAI and ghost CAI are shown in Table 4. We have included metal and sulfide contributions to both chondrule and matrix with 1:4 proportions, respectively, by considering their distributions in the real chondrites. The calculated molar proportions of each petrologic component (chondrule, matrix and CAI) are generally consistent with those in the real chondrites, with exception of CV chondrite (Efremovka) where the calculated proportion of chondrule is too high. Possibly the $\mathrm{Na}$ content of the average chondrule composition (average of only eight chondrules) for CV used in the calculation is not representative of the chondrules in Efremovka. If it is

${ }^{1}$ Here the molar proportion is defined as the total moles of the elements that constitute the mass of chondrules, divided by the total moles of the elements that constitute the bulk of chondrite. 


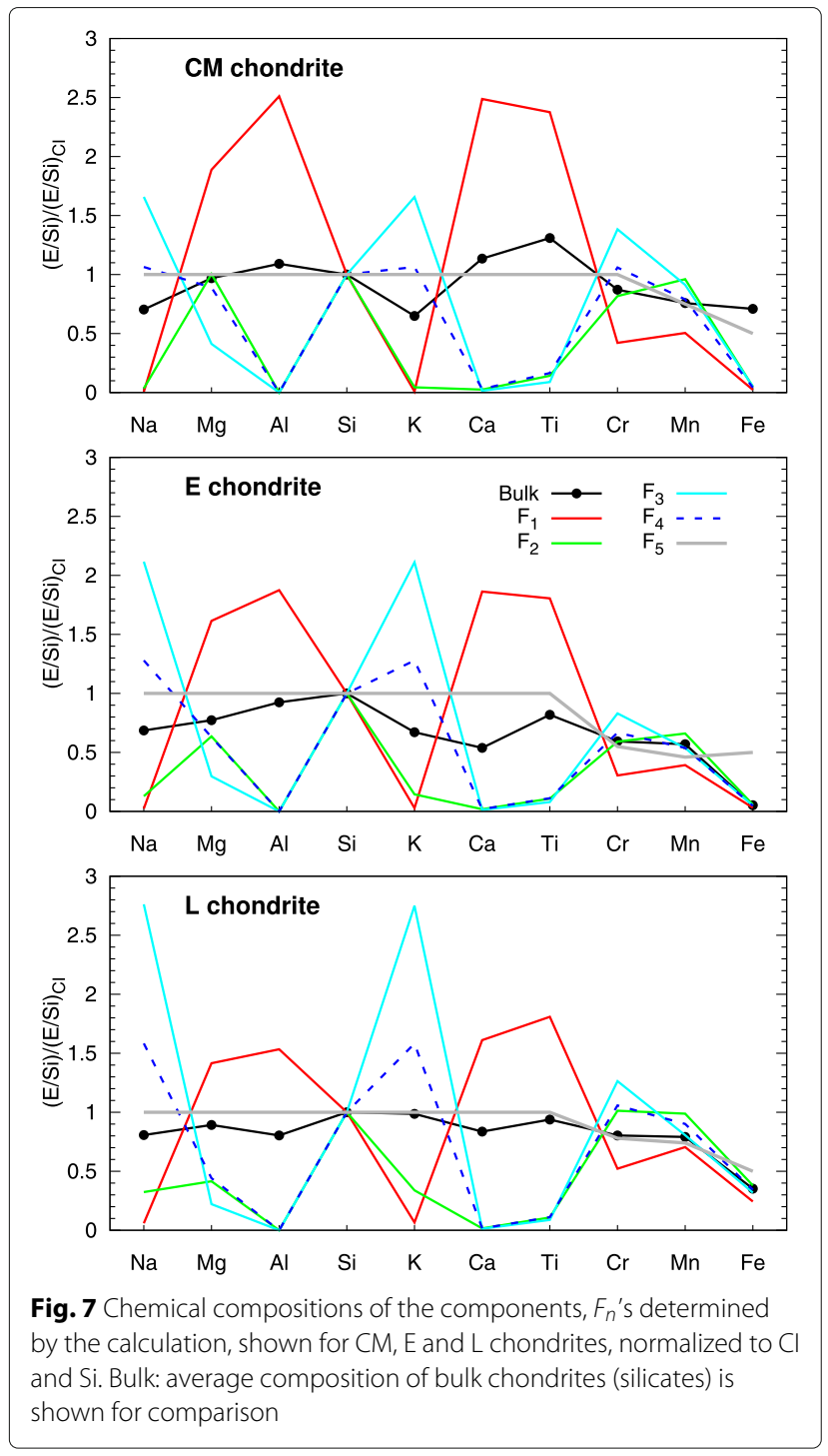

lower than the value used in the calculation, the chondrule proportion is decreased down to $70 \%$.

Figure 8 (bottom panel) shows molar proportions of CAIs and ghost CAIs in the bulk chondrites calculated. We identify three groups. CV, CM and E types contain only CAIs, while CO and LL contain both CAIs and ghost CAIs. $\mathrm{H}$ and $\mathrm{L}$ types are free of CAIs but contain small amounts of ghost CAIs. A very simple interpretation of the presence of CAIs and ghost CAIs in the chondrites is that CAIs invaded the chondrite formation zone after the chondrule forming process while ghost CAIs invaded before the chondrule formation. The coexistence of both CAIs and ghost CAIs in the same chondrite indicates the invasion of CAIs during the chondrule formation.

We find a concomitant increase in $f_{s}$ value among the three groups of chondrites. As shown in Fig. 5 (bottom panel) and Table 4, CV, CM and E chondrites are very low in $f_{s}, 0.03-0.04$; CO and LL have similar and higher $f_{s}$
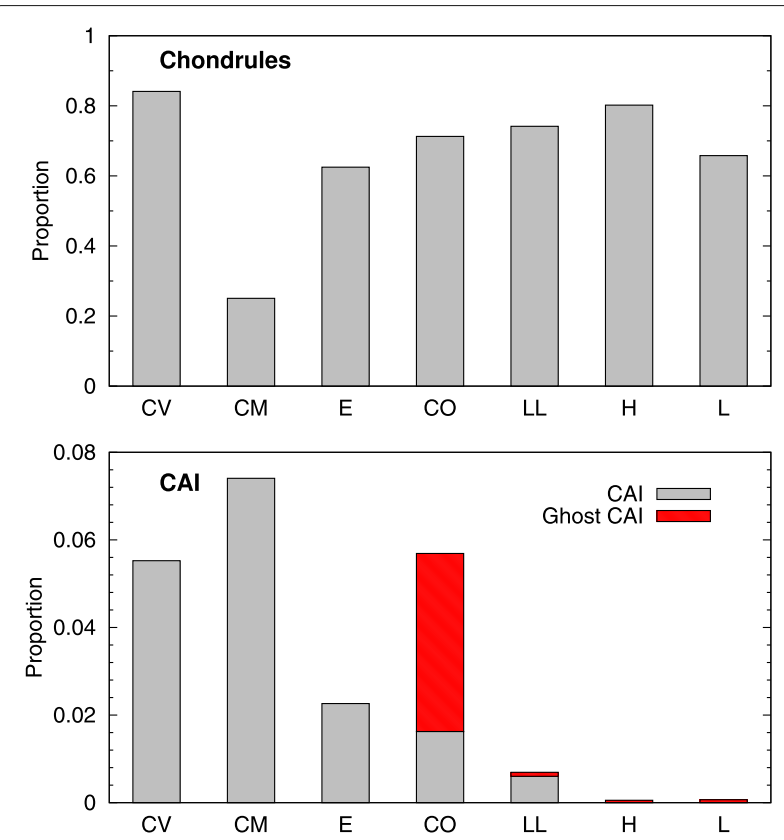

Fig. 8 Molar proportion of chondrules (top panel), and CAls + ghost CAls (bottom panel) in the bulk chondrite

values, 0.20 ; and $\mathrm{H}$ and $\mathrm{L}$ have the highest $f_{s}$ values, 0.22 0.27 . Thus we suspect that the invasion of CAIs is related with $f_{s}$, directly or indirectly, for some reason. This aspect will be focused in Section 4.1.

We come to the point what made the difference in chemical composition between different types of chondrites. The $f_{s}$ value most probably inherits the original redox state of the primordial dust. Hence different types of chondrites must have had different redox states in their source materials. This has a profound implication for the chemical evolution of PPD, as will be discussed in the next section.

The different VD values reached by MLB and the different proportions $F_{n}$ 's for different types of chondrites, on the other hand, must have reflected different physical conditions during the chondrule formation. Our current, jet-droplet \& vapor-cloud model is premature to give it a full consideration. We are developing a numerical code that includes necessary physics and boundary conditions (Hashimoto and Nakano, in preparation).

\subsection{Balance of the Elements Involved in the Chemical Fractionation}

We try to estimate the balance of the ten elements, 'used' and 'not used', in forming various types of chondrites from a duston having the $\mathrm{CI}^{\text {sil }}$ composition. For simplicity, we choose $\mathrm{CM}, \mathrm{CV}$ and $\mathrm{E}$ chondrites for this analysis, which do not require an admixture of ghost CAIs to the duston before the chondrule formation. $\mathrm{L}$ chondrite is also chosen because its minute amount of ghost CAI (a Ti-enriched, 
Ca-Ti oxide) is negligibly small. The data used in the calculation are (i) the compositions of the four components to be mixed and their proportions, $F_{n}$ 's $(n=1-4)$, (ii) the initial composition of duston, viz., $\mathrm{CI}^{\text {sil }}$ that takes into account the $f_{s}$ value of the chondrite, (iii) the VD\% for the average chondrule composition, (iv) the final VD\% reached by MLB, viz., a melted surface of the duston, which is taken twice as large as the VD\% for the average chondrule, and (v) the composition of the evolved MLB corresponding to the final VD\% (called 'evaporation residue' here). Note that the calculation excludes contribution of CAIs that later invaded into the chondrite forming zones.

One unknown factor is the production efficiency of jet-droplets ejected throughout the boiling process from
$\mathrm{VD}=0$ to the final VD\%. In our experiments (paper I), the run\# 138 and run\# SZ6 produced jet-droplets with a production efficiency of their integrated mass relative to the total mass lost (as a combined mass of jet-droplets and vapor) by the sample, 11.0 and $11.9 \mathrm{wt} . \%$, respectively. Some other runs produced jet-droplets in a range of 5-10 wt.\% relative to the initial masses of experimental charges. In the following calculation, we adopt a round number, 10 $\mathrm{mol} \%$ (mol\% instead of wt.\% because VD is also in mol\%) for the jet-droplet mass percentage (JDMP), defined as the molar fraction of total jet-droplets released during the boiling of a MLB relative to the initial MLB, for all types of chondrites for simplicity.

Figure 9 shows the proportions of the ten elements relative to their initial abundances in $\mathrm{CI}^{\text {sil }}$, which reside in
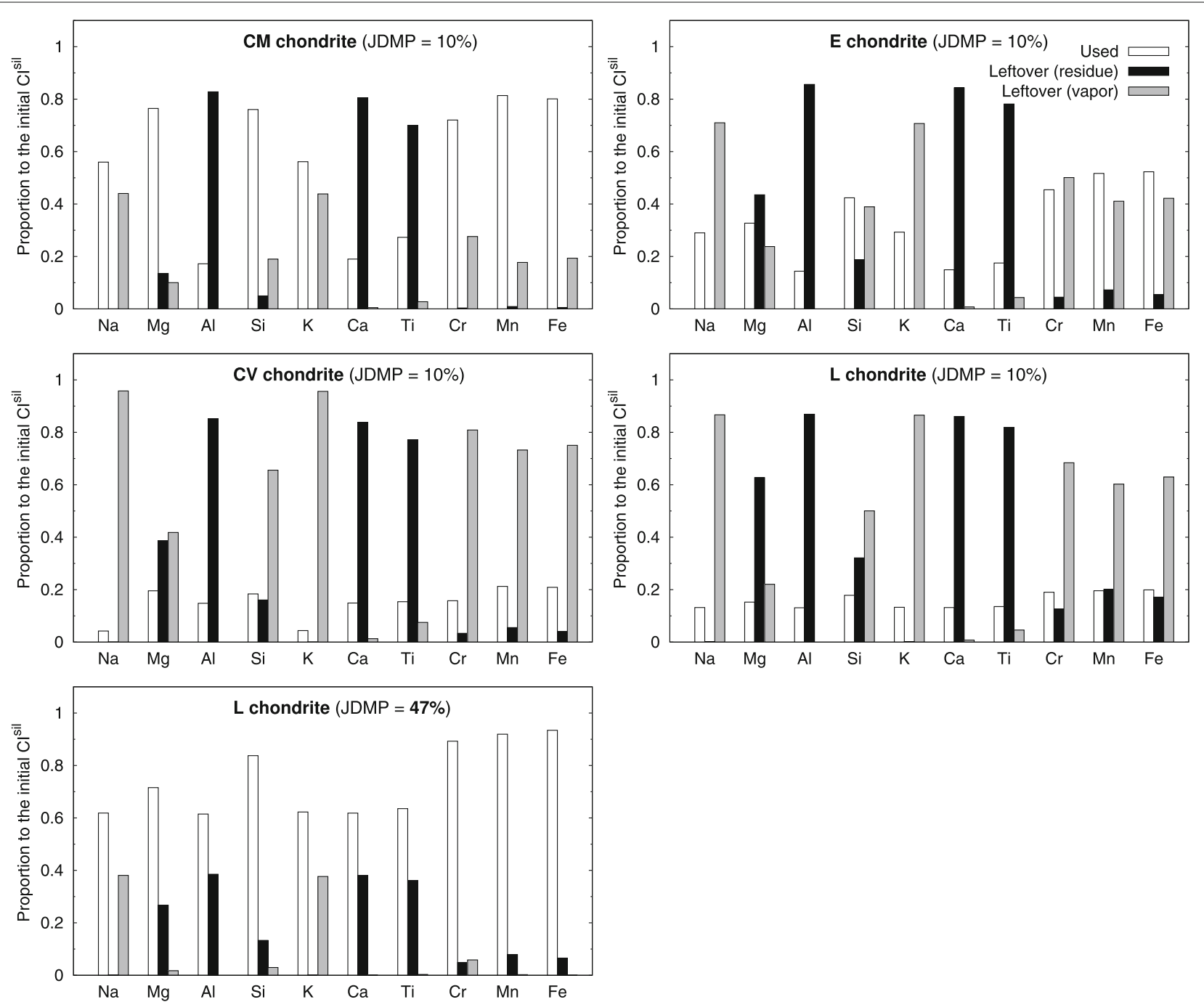

Fig. 9 Proportions of each of the ten elements relative to its initial abundance in $\mathrm{Cl}^{\mathrm{sil}}$, which reside in (A-1) the chondrite forming material (a total of $F_{1}$ through $\left.F_{4}\right),(A-2)$ the leftover residue of MLB that is not used in forming chondrules, and (A-3) the leftover vapor (or condensates) that is also not used in chondrule formation. Used: A-1 component. Leftover (residue): A-2 component. Leftover (vapor): A-3 component. JDMP: jet-droplet mass percentage 
(A-1) the chondrite forming material (a total of $F_{1}$ through $\left.F_{4}\right)$, (A-2) the leftover residue of MLB that is not used in forming chondrules, and (A-3) the leftover vapor (or condensates) that is also not used in chondrule formation. A JDMP of $10 \mathrm{~mol} \%$ relative to the initial MLB is assumed for all four chondrites. Note that the purpose of the present calculation is to reveal whereabouts of the elements included in the initial MLB after the chondrule formation. As shown in Fig. 9, A-1 ('Used') takes the elements in amounts necessary for forming the chondritic silicates, but additional mixing of the unprocessed $\mathrm{CI}^{*}\left(F_{5}\right.$ : the fifth component), CAI, metal and sulfide are required to form final bulk chondrites. A-2 is particularly enriched in the most refractory $\mathrm{Al}, \mathrm{Ca}$ and $\mathrm{Ti}$, and $\mathrm{Mg}$ comes second. A-3 is rich in $\mathrm{Na}$ and $\mathrm{K}$, but also contains considerable $\mathrm{Si}, \mathrm{Cr}, \mathrm{Mn}$ and $\mathrm{Fe}$.

The CM chondrite is most efficient in forming a chondrite mass from the MLB, while L, CV and E chondrites must dump $70-80 \%$ of the initial mass as residue and vapor. If the constraint of JDMP of $10 \mathrm{~mol} \%$ is removed, a maximum possible JDMP can be calculated; 11.3, 18.0, 42.4 and $47.0 \mathrm{~mol} \%$ for CM, E, CV and L chondrite, respectively. Above these percentages, mass balance is not maintained. On the other hand, a minimum JDMP is not limited down to 0\%. Fig. 9 (bottom panel) show the case for L chondrite with a JDMP of $47.0 \mathrm{~mol} \%$ for example. However, such a presentation with a JDMP much larger than 10 mol\% may not be strictly valid because our calculation is based on the assumption that a mass loss of MLB by ejection of jet-droplets is minimal.

The important point is that the two components, A-2 and A-3, described above need to be separated from the component A-1 physically; otherwise chemical fractionations in chondrites are not completed. In Section 4.2, we hypothesize a mechanism how the three components were separated and where they were destined for.

\section{Discussion}

\subsection{Constraints to the Chemical Evolution of PPD}

Following the quantitative analyses in configuring bulk chemistry of various types of chondrites (Section 3), we distinguish chondrites between three generation groups in accord with the order of events, CAI incorporation versus chondrule formation. G-1: CAIs were incorporated into chondrite-forming components 'after' the chondrule formation event, as represented by $\mathrm{CV}, \mathrm{CM}$ and $\mathrm{E}$ chondrites. G-2: CAIs were incorporated 'during' the chondrule formation, like CO and LL. G-3: CAIs were incorporated 'before' the chondrule formation, like $\mathrm{H}$ and $\mathrm{L}$. If the CAIs formation and their subsequent migration to the outer disk had occurred during a limited period in the PPD evolution, it can be used as a time pivot for whatever processes that are related to the CAIs. Thus the three generations of chondrites recognized above are interpreted as reflecting a formation sequence in the order, G-1 through G-3, as to their chondrule forming events, on condition that the chondrule forming location, viz. its radial distance from the protostar, is fixed.

In Sections 4.2 and 4.4.3, we propose the snowline in the disk as an ideal place for chondrule formation. Thus, if the snowline moved radially in a time scale comparable to or shorter than the CAI migration timescale, the order (G-1 through G-3) might not represent the formation sequence. Here, we continue our investigation by assuming that the snowline was relatively stationary in the radial direction during the period of CAI migration.

As indicated in Section 3.5, $f_{s}$ (a redox indicator of primordial dust or duston) ascends in the order, $\mathrm{CV}, \mathrm{CM}, \mathrm{E}$, $\mathrm{CO}, \mathrm{LL}, \mathrm{H}$ and $\mathrm{L}$ chondrites. This is exactly the same order as G-1 $\rightarrow$ G-2 $\rightarrow$ G-3.

With these facts and the assumption, together with other observational information of chondrites and comets, we attempt to make a prediction about a possible evolutionary sequence of primordial PPD materials.

\subsubsection{Evolution in redox state}

The increase in $f_{s}$ with the order G- $1 \rightarrow$ G- $2 \rightarrow$ G- 3 suggests that the redox state of primordial dust or dustons evolved towards oxidizing with time. We speculate when, where and how it happened.

In their chemical network model of PPD, Walsh et al. (2015) have indicated that gas phase reactions dominate in the inner disk $(\leq 10 \mathrm{au})$ because of high temperatures and that the reaction of atomic $\mathrm{O}$ (produced by photodissociation of $\mathrm{CO}$ ) with $\mathrm{OH}$ (produced by reaction $\mathrm{O}+\mathrm{H}_{2}$ ) proceeds to form molecular oxygen,

$$
\mathrm{O}+\mathrm{OH} \rightarrow \mathrm{O}_{2}+\mathrm{H}
$$

Furuya et al. (2013), in their reaction network model of PPD, have suggested that at 10 au ( $60 \mathrm{~K})$ ultra-fine ice grains $(\sim 0.1 \mu \mathrm{m})$ are continuously supplied to the disk surface by turbulent vertical mixing and destroyed to atoms by stellar UV photons and X-rays, which in turn come back to the disk midplane by turbulence. Atomic oxygen is then converted to molecular oxygen by reaction 2, which becomes a predominant $\mathrm{O}$-bearing species in the disk, given enough time ( $\left.>10^{5} \mathrm{ys}\right)$.

We propose that the molecular oxygen was radially transported by turbulent diffusion and made the disk gradually oxidized inward as well as outward. This picture qualitatively matches the chronological order of the generation groups of chondrites, G-1 through G-3.

As an alternative, the cometary $\mathrm{O}_{2}$ can be a source of oxidizer in PPD. The comet 67P/ChuryumovGerasimenko is known to have kept producing a neutral gas coma with unusually abundant $\mathrm{O}_{2}$ molecules 
$\left(\mathrm{O}_{2} / \mathrm{H}_{2} \mathrm{O}=0.01-0.1\right)$ (Bieler et al. 2015) during its period of observation. In their model calculations of the chemical evolution of material from the pre-stellar core to the midplane of PPD, Taquet et al. (2016) and Mousis et al. (2016) have investigated the yield of $\mathrm{O}_{2}$ ice in cometary ice inventories and concluded that the high abundance of $\mathrm{O}_{2}$ in 67P, if produced solely as $\mathrm{O}_{2}$ ice by radiolysis, is only adequately explained as having formed in the parent cloud. Eistrup and Walsh (2019) have found a sweet spot parameters for production of $\mathrm{O}_{2}$ ice in the pre-solar nebula midplane at a level matching those in $67 \mathrm{P}$ and $1 \mathrm{P} /$ Halley, but preferred a primordial origin in the parent cloud, having considered a sensitivity of such a result to the assumed chemical parameters.

If true, the $\mathrm{O}_{2}$-rich cometary materials such as $67 \mathrm{P}$ and $1 \mathrm{P}$ might be a source for dustons that formed the generation group G-3 (viz., $\mathrm{H}$ and $\mathrm{L}$ chondrites), while the $\mathrm{O}_{2}$-free or -poor cometary materials like most other comets could be a source for the generation group G-1 (viz., CV, CM and E chondrites) and perhaps G-2.

Whichever mechanism of $\mathrm{O}_{2}$ enrichment in PPD is true, the $\mathrm{O}_{2}$ molecules must have reacted with metal and sulfide embedded in the primordial dust to form iron oxides.

\subsubsection{Evolution in $D / H$ ratio}

Figure 10 shows $\mathrm{D} / \mathrm{H}$ ratios in various substances in our solar system and extrasolar environments. $\mathrm{CV}$ and $\mathrm{CM}$ chondrites which have the lowest $f_{s}$ values and hence are the most reduced types in our study, show the lowest $\mathrm{D} / \mathrm{H}$ ratios $\left(<1 \times 10^{-4}\right)$ among the chondrites (Alexander et al. 2012). LL chondrites which have a higher $f_{s}$ value in our study, show a high $\mathrm{D} / \mathrm{H}$ ratio $(\sim 3 \times$ $10^{-4}$ ) (Alexander et al. 2012). $\mathrm{R}$ chondrites, which are not included in our study due to the lack of composition data, is known as the most oxidized type and have the highest $\mathrm{D} / \mathrm{H}$ ratio $\left(7.26 \times 10^{-4}\right)(\mathrm{McCanta}$ et al. 2008). The comet 67P/Churyumov-Gerasimenko, in spite of its being a J. F. Comet, have the highest $\mathrm{D} / \mathrm{H}$ ratio $\left(5.3 \times 10^{-4}\right)$ among the comets ever observed (Altwegg et al. 2015). This comet contains unusually abundant $\mathrm{O}_{2}$ molecules as described above. All these evidence points to a strong correlation between the high oxidation state and the high $\mathrm{D} / \mathrm{H}$ ratio.

The water ice is mostly responsible for the $\mathrm{D} / \mathrm{H}$ ratios of chondrites and comets except some contribution of the organic hydrogen (Alexander et al. 2012). Interstellar ices show the highest $\mathrm{D} / \mathrm{H}$ ratio of $\sim 10^{-2}$ in the galaxy (Fig. 10). It is postulated that $\mathrm{H}_{2} \mathrm{D}^{+}$is preferentially produced by ion-neutral reactions in a molecular cloud core and is subsequently turned into ices in the coldest $(\sim 10 \mathrm{~K})$ environment (Cleeves et al. 2014). All matters including gas, ice, silicate and organics in PPD come from a molecular cloud core (e.g., Williams and Cieza 2011). In fact, the $\mathrm{D} / \mathrm{H}$ ratios of all the solar system materials lie between $10^{-2}$ and $2 \times 10^{-5}$ (that of the Sun) as shown in Fig. 10. This implies that some amount

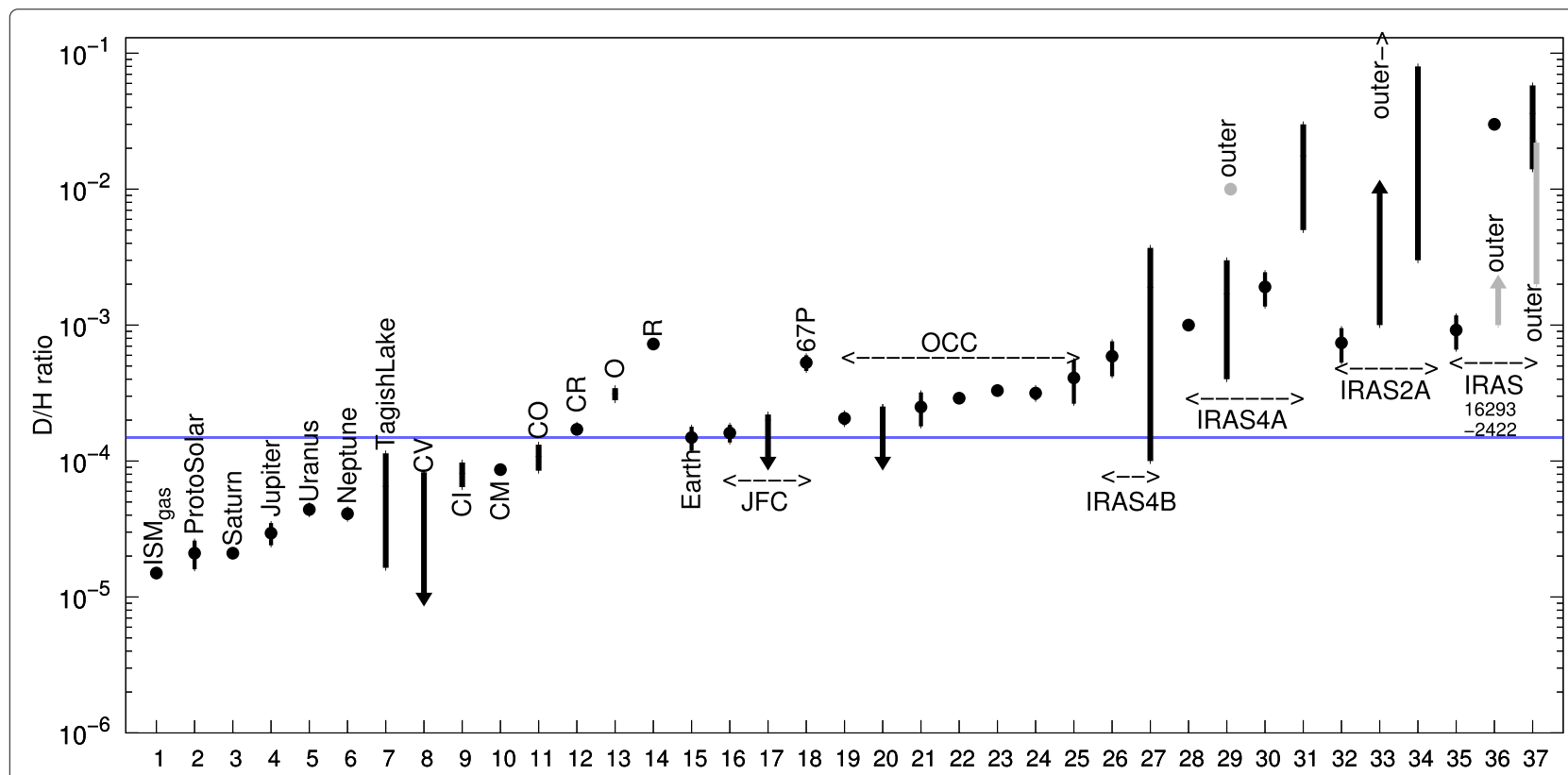

Fig. $10 \mathrm{D} / \mathrm{H}$ ratios of various solar system and extrasolar substances. Data and their references noted by numbers 1-37 are shown in Appendix B. Blue-line represents the D/H ratio of Bulk Earth (15). Numbers 7-14 are chondrites. JFC: Jupiter family comets. OCC: Oort cloud comets. For other details, see Appendix B 
of interstellar ice survived the formation of the solar system and was incorporated into planetesimal bodies (Cleeves et al. 2014).

Furuya et al. (2013) proposed a model that explains the decrease in $\mathrm{D} / \mathrm{H}$ ratio of water ice in PPD. Depending on the temperature in the midplane (either $<40 \mathrm{~K}$ or $>60 \mathrm{~K}$ ), atomic oxygen is either transformed into water ice (regeneration of water ice) by reaction with adsorbed hydrogen on the ice surface, or into molecular oxygen in the gas phase reaction such as reaction 2. Given enough time ( $>10^{5} \mathrm{ys}$ ), the regeneration of water ice reduces the $\mathrm{D} / \mathrm{H}$ ratio of water ice (Furuya et al. 2013). In the latter case, on the other hand, the initial $\mathrm{D} / \mathrm{H}$ ratio of water ice tends to be maintained in exchange for depletion of the water ice, and the disk gas becomes gradually loaded with $\mathrm{O}_{2}$ as described above.

The role of atomic oxygen, however, is not one-sided since both ice-regeneration and $\mathrm{O}_{2}$-formation should concur (Furuya et al. 2013) and it is a matter of bias depending on the midplane disk temperature. We postulate that the disk conditions had changed with a timescale $\sim 10^{5}$ years in such a way that the average midplane temperature increased.

The reason why the increase in the $\mathrm{D} / \mathrm{H}$ ratio of chondrites and comets has a good correlation with the increase in their oxidation states is easily understood because both require the production of free oxygen in PPD.

\subsubsection{Chronology of chondrules and CAls}

Here we examine whether the generation sequence of chondrites, G-1 $\rightarrow \mathrm{G}-2 \rightarrow \mathrm{G}-3$, is consistent with the chronological information of chondrules and CAIs, on condition that the chondrule forming location, viz., its radial distance from the protostar, is fixed as explained in Section 4.1.

CAIs typically have excess ${ }^{26} \mathrm{Mg}$, a trace of extinct radionuclide ${ }^{26} \mathrm{Al}$ at the canonical level of initial ${ }^{26} \mathrm{Al} /{ }^{27} \mathrm{Al}$ $\sim 5 \times 10^{-5}$, while CAIs with less or no excess ${ }^{26} \mathrm{Mg}$ are also abundant (see review by MacPherson et al. 1995). Chondrules, on the other hand, generally have no excess ${ }^{26} \mathrm{Mg}$, while some have initial ${ }^{26} \mathrm{Al} /{ }^{27} \mathrm{Al} \sim 8 \times 10^{-6}$ (e.g., Hutcheon and Hutchison 1989; Russell et al. 1996). With the half-life of ${ }^{26} \mathrm{Al}=7.3 \times 10^{5} \mathrm{yr}$, it has been interpreted that there is an age difference between CAIs and chondrules of $\sim 2.5 \mathrm{Myr}$ or more. It looks like an immediate contradiction because the chondrule formation of the generation group G-1 (viz., CV, CM and E) occurred before the invasion of CAIs. The generation group G-3, on the other hand, requires that it should have taken CAIs for $>2.5 \mathrm{Myr}$ to travel from its formation zone (perhaps the inner rim of the disk; e.g., Dullemond et al. 2007) to the zone of chondrule formation $(\sim 3$ au if it coincides with the asteroid belt) because the chondrule formation of the group G-3 (viz., H and L) occurred after CAI invasion.
Considering the mass accretion rate of T-Tauri stars typically of $\sim 10^{-8} \mathrm{M}_{\odot}$ per year (Hartmann et al. 2016), such an age difference is comparable to or longer than the time scale for dissipation of the gas disk, and thus unlikely.

Our result implies an irrefutable possibility that the ${ }^{26} \mathrm{Al}$ ages for chondrules (Davis and McKeegan 2014, references therein) are not real. In our investigation, chondrites except for $\mathrm{CV}, \mathrm{CM}$ and $\mathrm{E}$ types contain ghost CAIs, indicating that chondrules formed from silicate liquids made from a mixture of primordial CI-like dust and invading CAIs. Even if the primordial dust contains no ${ }^{26} \mathrm{Al}$, chondrules should have ${ }^{26} \mathrm{Al}$ as a result of mixed CAIs in the silicate liquid. In fact, $\mathrm{CO}$ and LL chondrites for which the invasion of abundant ghost CAIs are implied from the present analysis contain many chondrules having excess ${ }^{26} \mathrm{Mg}$, and this is particularly so for their unmetamorphosed types (3.0 or 3.1) (Kita et al. 2000; Mostefaoui et al. 2002; Kunihiro et al. 2004; Kurahashi et al. 2008). Chondrules with high $\mathrm{Al}$ contents are preferentially analyzed because of their high precision in determination of ${ }^{26} \mathrm{Al} /{ }^{27} \mathrm{Al}$ (e.g., Kita and Ushikubo 2012). As a matter of fact, the high- $\mathrm{Al}$ chondrules exhibit highest ${ }^{26} \mathrm{Al} /{ }^{27} \mathrm{Al}$ values, $\sim 1 / 5$ of the canonical CAI value (Davis and McKeegan 2014), reinforcing our prediction. We presume that chondrules with high concentrations of live ${ }^{26} \mathrm{Al}$ simply represent jet-droplets from portions of the silicate liquid in MLB, that is inhomogeneously mixed with large CAIs. We conclude that the ${ }^{26} \mathrm{Al}$ chronology for chondrules is subject to a serious problem in that their real ages are indeterminable because of the pre-mixing of CAIs which are high in $\mathrm{Al}$ and ${ }^{26} \mathrm{Al}$.

Connelly et al. (2012) and Bollard et al. (2017) have shown that the ages of six among twenty-two chondrules measured with the $\mathrm{U}$-corrected $\mathrm{Pb}-\mathrm{Pb}$ dating method agree with the average age of four CAIs (4567.30 \pm 0.16 Myr) within errors. With this result they claim that chondrules and CAIs are coeval, and that the assumption of the uniform distribution of ${ }^{26} \mathrm{Al}$ in PPD is wrong. If they are coeval, the time interval for chondrule formation between the group G-1, G-2 and G-3 could be as short as $0.16 \mathrm{Myr}$, although only six chondrules show such a sign and only four data are available for CAIs ages. Their results, however, appear contradictory because the ages of thirteen chondrules from NWA 5697 (L3) span between 4563.64 and $4567.61( \pm 0.50)$ Myr, viz., $\sim 4$ Myr interval. It seems improbable that a single chondrite could reserve chondrules that had been continuously produced in PPD for as long as $4 \mathrm{Myr}$.

$\mathrm{The} \mathrm{Pb}-\mathrm{Pb}$ chronology for chondrules appears to be free of the problem of CAI mixing unlike ${ }^{26} \mathrm{Al}$, but the wide age span of chondrules in a single chondrite cast a doubt on using it as a constraint on the timing of chondrule formation. We explore a little more about the age data. The four CAIs used for determination of their $\mathrm{Pb}-\mathrm{Pb}$ ages 
(Bollard et al. 2017) are limited to those in CV chondrites (three from Efremovka and one from Allende). In our study, CV in the generation group G-1 is actually Efremovka itself. The U-corrected $\mathrm{Pb}-\mathrm{Pb}$ method analyzed only two chondrules that belong to CV chondrites (Connelly et al. 2012). They are Allende chondrules, one of which shows $4567.32 \pm 0.42 \mathrm{Myr}$ in good agreement with the CAI age although another is a Myr younger. Thus the chondrules and CAIs in CV chondrites (viz., Efremovka and Allende), as far as the existing data concerned, agree in their ages within analytical errors except one chondrule. This is consistent with our observation of the generation G-1 in which chondrules formed prior to the invasion of CAIs, as long as the time needed for CAIs to migrate to the zone of chondrule formation is short. We therefore suggest that a comparative chronology should be made on chondrules and CAIs that belong to the same chondrite or at least to the same chemical type of chondrites.

\subsection{Completion of the Chemical Fractionations}

Chemical fractionations in chondrites are not completed until the leftover refractory residue of MLB and the leftover volatile vapor (or condensates) are separated from the chondrite-forming ingredients, viz., chondrules and matrix.

The physical separation is only possible through aerodynamic actions operating on objects with different sizes. The leftover evaporation residue is a few-mm thick, oncemolten, surface layer of a large duston with the radius $R>$ $0.01 \mathrm{~m}$. Bodies with $R$ in the $0.01-10 \mathrm{~m}$ range lose their angular momentum per unit mass much faster compared to bodies of other sizes, due to their differential motions against the gas. According to Weidenschilling (1977), a body with $R=1 \mathrm{~m}$ (solid $\rho \sim 3 \mathrm{~g} \mathrm{~cm}^{-3}$, gas $\rho \sim 10^{-9} \mathrm{~g}$ $\mathrm{cm}^{-3}$ and $\mathrm{T} \sim 300 \mathrm{~K}$ ) would migrate to the sun in $\sim 50 \mathrm{yr}$ per au, while it takes $\sim 5000 \mathrm{yr}$ per au for $R=0.01$ or 10 $\mathrm{m}$ (for the same parameters). On the other hand, grains smaller than chondrules $(R \leq 0.001 \mathrm{~m})$ take $\geq 10^{5}$ yr per $\mathrm{au}$ (ibid) and should remain in the chondrule formation zone for a long time.

We propose that dustons were trapped in a large-scale vortex in the protoplanetary disk and that the chondrule formation took place inside the vortex immediately after their entrapment. There the Coriolis force is at work, which pushes dustons of appropriate size, $0.01-10 \mathrm{~m}$ in radius, to the center of the vortex in a few rotation periods (e.g., Tanga et al. 1996; Chavanis 2000). The result would be that small objects such as chondrules and finer dust are left near the outer edge of the vortex. Small grains with diameter $<1 \mathrm{~cm}$ tend to bail out to the outside of the vortex due to turbulent diffusion (Chavanis 2000). Therefore chondrules must grow in size by attaching fine dust rather efficiently (Beitz et al. 2012). Once chondrule-cored nodules and their multiple clusters grow in size $>1 \mathrm{~cm}$, they would run after dustons but at a slower pace. In this way we expect three populations being separated from one another as a result of aerodynamic effects: (Pop-1) the chondrule-cored nodules and their clusters that follow the dustons much later in time, (Pop-2) the dustons with refractory residues that settle in the center of the vortex in a short time scale, and (Pop-3) the fine dust, a mixture of the volatile-rich condensate and the primordial CI-like dust, which escape from the vortex. The three populations correspond to the three components that are produced in the chemical fractionation process during the chondrule formation, (A-1), (A-2) and (A-3) as described in Section 3.6, except that Pop-2 consists of A-2 and a nonmelted portion of dustons, and Pop-3 is a mixture of A-3 and the primordial dust.

We discuss the two populations that remain in the vortex. The dustons (Pop-2) are so effectively trapped into the center of vortex (e.g., Barge and Sommeria 1995; Fromang and Nelson 2005; Surville et al. 2016) that a gravitational instability is triggered to generate a large planetesimal (e.g., Tanga et al. 1996). A swarm of the late-arriving, chondrule-cored nodules, viz., Pop-1, would be gravitationally attracted by the planetesimal and accumulate onto its surface, forming a layer of 'chondrite'. As a result the chemical fractionation is completed because the chondrite is spatially segregated from the dustons that form the core of the planetesimal and represent most of its mass. Vortices may not be only attractor of dustons. Any mechanism that attract dustons preferentially (e.g., a dustloaded ring: Surville et al. 2016) could provide a place for forming planetesimals and late veneers of chondrites around them.

As a logical consequence we cast doubt on a hypothetical, meteorite (or chondrite) parent body. The petrologic types (from 3.0 to 7 ) of chondrites have long been believed as representing thermal metamorphic grades which reflected depths of the sources where the chondrites came from after destruction of the parent body. Rubin (2003, 2004) and Rubin and Wasson (2011) found that the common occurrence of shocked and annealed ordinary and enstatite chondrites is consistent with collisional heating being a major mechanism responsible for metamorphosing those chondrites. Friedrich et al. (2014) found that ${ }^{26} \mathrm{Al}$ was too depleted to have caused high degrees of thermal metamorphism and partial melting of LL7 chondrites and that heating due to impact is the most likely heat source for the recrystallized chondrites. We'd like to point out that the outermost layers of planetesimals should have been most prone to shocks and disruption by impacts of other bodies. Disrupted pieces may have accumulated on their original planetesimal surface or formed independent rubble piles that were loosely tied with weak gravity, as seen in extant asteroid belts (Walsh 2018). The third population (Pop-3) may have been further 
mixed with a CI-like dust in the disk and diluted to an insignificant concentration. Recent rendezvous missions to comets, however, revealed high concentrations of crystalline silicates in cometary comas, which are highly enriched in $\mathrm{Na}$ and Si relative to Mg (Schulz et al. 2015; Engrand et al. 2016; Bardyn et al. 2017). The enrichment of volatile $\mathrm{Na}$ (and $\mathrm{K}$ ), and $\mathrm{Si}$ relative to $\mathrm{Mg}$ is exactly a feature of the volatile condensates in the third population (Fig. 9). The third population may also give a hint to the origin of GEMS (Keller and Messenger 2011), UCAMMs (Dobrica, et al. 2012) and CPMMs (Noguchi et al. 2015), all of which are suspected to have genetic links to comets.

\subsection{Comparison with the Equilibrium Condensation Model}

Having discussed our new ideas on the origin of the chemical fractionations in chondrites, we come to a point where we could compare our model with those developed by Anders and his group. As described in Section 1, their model basis, viz., the equilibrium condensation of chondritic materials, has lost its reasoning. However, it might be beneficial for us and all to do this since essential points would be clarified. In addition to the chemical aspects which will be described below, there are several physical difficulties attached to their model. 1) The grain size of particles that condense from a gas of low density should be too small to be separated from the gas (Weidenschilling 1977), making the fractionation absolutely ineffective. 2) No reason is given for any particular temperatures at which they claim the fractionations should occur. 3) Chondrules, the most abundant constituent of chondrites, is not taken into consideration.

\subsubsection{Lithophile element fractionation}

We refer to the major element or refractory lithophile element fractionations in chondrites (Larimer and Anders 1970; Larimer 1979). Larimer and Anders (1970) have proposed that a partial loss of high-temperaturecondensation minerals at appropriate temperatures and a subsequent condensation of minerals which followed from the remaining gas reservoir could explain major chemistry of ordinary and enstatite chondrites, starting from the CI-composition gas. They derived chemical compositions (for the five major elements, $\mathrm{Si}, \mathrm{Mg}, \mathrm{Ca}, \mathrm{Al}$ and $\mathrm{Ti}$ ) similar to those of bulk ordinary (and enstatite) chondrites, by subtracting 15 (25), 23 (45) and 40 (60) $\%$ of $\mathrm{Si}, \mathrm{Mg}$ and $\mathrm{Ca}$ (same for $\mathrm{Al}$ and $\mathrm{Ti}$ ), respectively, from the CI composition. Those minerals were identified as typical CAI minerals and forsterite. As they pointed out, these percentages are examples and other combinations are possible. Larimer (1979), based on his analysis on the condensation track of major elements and average compositions of ordinary and enstatite chondrites, have proposed that the bulk mineral chemistry to be subtracted from the CI composition was uniquely determined. His estimates of mass subtraction are not so different from those by Larimer and Anders (1970).

In order to explain the bulk chemical compositions of CII and CIII (now classified as CM for CII, and CO and CV subtypes for CIII), Larimer and Anders (1970) inexplicitly and Larimer (1979) explicitly suggested the addition of CAI minerals in appropriate proportions to the CI composition.

Larimer and Anders (1970) concluded that the chondrule formation had nothing to do with the lithophile element fractionations, and that chondrules were simply molten droplets of the existing solids.

An essential difference between our model and their model is that we consider the chondrule formation and the subsequent dynamical processes as a primary driving force for the lithophile element fractionation, while they do not. Let us recapitulate our model for the sake of comparison.

As shown in Fig. 9, the initial CI-like material, duston, is processed into three kinds of materials as a result of chondrule formation process in the vapor cloud that forms around it. The chondrule- and matrix-forming components, $F_{1}$ through $F_{4}$ in the calculation, when combined, are depleted in refractory elements such as $\mathrm{Al}, \mathrm{Ca}$ and Ti relative to $\mathrm{Mg}$ and Si (Fig. 9; 'Used'). As a result, not only enstatite and ordinary chondrites but also carbonaceous chondrites suffer from depletion in the refractory elements once. The difference among these chondrites is the amount of CAIs which invaded into a zone of chondrite formation. For carbonaceous chondrites, refractory elements (in the form of CAIs) invaded and mixed with chondritic components in amounts more than compensating the lost fraction during the chondrule formation. Enstatite chondrites incorporated Al-rich CAIs in quantity too small to compensate the largest depletion in the refractory elements caused during the chondrule formation. Ordinary chondrites ( $\mathrm{L}$ and $\mathrm{H}$ types) incorporated no CAI except for a trace of ghost CAI, thus remained depleted in the refractory elements. Ordinary chondrites (LL type) incorporated both CAI and ghost CAI, but their combined mass was too small to compensate the depletion in the refractory elements. Such is a brief explanation in our model for the lithophile element fractionations in chondrites.

To complete the chemical fractionation, however, the leftover residue which is enriched in the refractory elements (Fig. 9; 'Residue'), must be removed from the chondrule-forming zone. This is automatic because the residue is a once-molten surface layer of a large duston which subsequently falls into the center of a large-scaled vortex by the aerodynamic process. The equilibrium condensation model, on the other hand, has no means to remove the high-temperature condensates from their 
formation zone since they are ultra-fine-grained particles that strongly couple with the gas.

The removal of excess volatiles that are not incorporated into chondrite-forming materials (Fig. 9; 'Vapor') is also automatic in our model because the vapor condensates, fine-grained in nature, would effectively move away from the vortex by turbulent diffusion. The removed volatiles in the form of low-temperature condensates must include not only $\mathrm{Na}$ and $\mathrm{K}$ but also highly volatile elements (e.g., $\mathrm{Pb}, \mathrm{Bi}$, In, Tl). Therefore, our model does not have to invoke any extra chemical fractionation process such as accretion-temperaturecontrolled fractionation of volatiles proposed by Keays et al. (1971) and Laul et al. (1973) (viz., Chem. fract. in meteorites-IV \& -VI).

\subsubsection{Redox state and metal-silicate fractionation}

Different types of chondrites have different redox states of $\mathrm{Fe}$ and different total Fe contents (Urey and Craig 1953). Fe exists in meteorites typically with three different states: oxides (mostly as $\mathrm{FeO}$ ) in silicates, metals and sulfides. In the latter two, $\mathrm{Ni}$ and $\mathrm{Co}$ are invariably included as minor elements, which under most redox conditions are not oxidized. Larimer and Anders (1970) examined concentrations of $\mathrm{Fe}$ and $\mathrm{Ni}$ in various chondrites and ascribed the depletion in total $\mathrm{Fe}$ in $\mathrm{L}$ and LL chondrites as opposed to $\mathrm{H}$ and $\mathrm{C}$ chondrites, to the metal-silicate fractionation that had occurred between $\sim 1050 \mathrm{~K}$ and $680 \mathrm{~K}$ (temperature for FeS formation) before accumulation of chondritic materials into large bodies.

Larimer and Anders regarded the redox state of minerals in chondrites as reflecting their final equilibration temperatures with the nebular gas. In the equilibrium condensation model of high temperature nebular gas with the solar elemental abundances, Fe metal condenses at $\sim 1450 \mathrm{~K}$ at $10^{-3}$ bar in total pressure (Grossman 1972) and at $\sim 1280 \mathrm{~K}$ at $10^{-5}$ bar (Wood and Hashimoto 1993). No other Fe-bearing phase appears down to $\sim 900$ $\mathrm{K}$ where a trace of Fe metal is oxidized and accommodated into Mg-silicates (forsterite and enstatite), but it is not until $700 \mathrm{~K}\left(10^{-3}\right.$ bar $)$ or $600 \mathrm{~K}\left(10^{-5}\right.$ bar $)$ where olivine takes $\mathrm{FeO} /(\mathrm{MgO}+\mathrm{FeO})=0.05$, which is close to the $\mathrm{FeO}$ concentration in enstatite in E-chondrites and in forsterite in a reduced type of $\mathrm{CV}$ chondrite, viz., Efremovka. In order to oxidize $\mathrm{Fe}$ to a level of $\mathrm{FeO} /(\mathrm{MgO}+\mathrm{FeO}) \sim 0.2$ in olivine typically in ordinary chondrites, temperatures as low as $520 \mathrm{~K}$ is required (Wood and Hashimoto 1993).

We point out a contradiction between the above two arguments. The $\mathrm{Fe} / \mathrm{Ni}$ molar ratios of bulk chondrites are 16.6 for $\mathrm{H}, 18.6$ for L and 19.3 for LL (Jarosewich 1990), while the Fe/Ni ratio in CI chondrites is 18.1 (Lodders 2003). Comparison of the temperature range for the metal-silicate fractionation with those for oxidation of $\mathrm{Fe}$ metal, however, implies that the metal-silicate fractionation occurred before the oxidation. If so, the $\mathrm{Fe} / \mathrm{Ni}$ ratio of chondrites should keep 18.1 if CI was their starting composition. Hence their model with respect to the redox states and metal-silicate fractionation bears an internal inconsistency.

The Fe/Ni ratios of bulk chondrites higher than 18.1 indicates that the oxidation of Fe occurred before the metal-silicate fractionation. L and LL chondrites must have lost a significant mass of metal before their accretion to large bodies. The lower Fe/Ni ratio of $\mathrm{H}$ chondrites relative to $\mathrm{CI}$, on the other hand, suggests a gain of excess metal or a loss of silicate before their accretion.

In contrast with Larimer and Anders (1970), the discussions made in Sections 3 and 4.1.1 strongly argue for the possibility that the redox state of $\mathrm{Fe}$ in the primordial dust was predetermined before the chondrule formation process and that it evolved with time from reducing to oxidizing, corresponding to the generation sequence of chondrites, G-1 through G-3. This is consistent with the order of the events indicated by the $\mathrm{Fe} / \mathrm{Ni}$ ratios of chondrites, that the oxidation is followed by the metal-silicate fractionation, since the latter should have occurred during or after the chondrule formation in our model.

\subsection{In Search of Possible Heat Sources}

Our model assumes that the surface of duston was heated above their boiling temperature with some external source of heat. Let us scrutinize possible heating mechanisms in PPD suggested for chondrule formation. Rubin (2000) has considered ten constraints that are related with petrologic and geochemical features of chondrules and made a check list for sixteen heating mechanisms with these constraints (figure 5 in his paper). He has pointed out the three mechanisms being consistent with all the constraints: nebular lightning, magnetic reconnection flares, and gas dynamic shock waves.

We introduce two lines of evaluation procedures apart from his. The first line has to do with the coexistence of chondrules with a matrix. In chondrites the finegrained matrix materials fill interstices between chondrules. Their abundance in volume ranges from 20 to $80 \%$, depending on the type of chondrites. They are free of melting process and generally rich in volatiles including organic materials and water, being characteristic of lowtemperature origin. It means that any heating mechanism which violates the presence and features of the matrix materials is not appropriate for chondrule formation. The second line is the consistency with our chondrule and chondrite formation model, and is considered after the first line.

We apply our evaluation procedures to the following heating mechanisms from Rubin (2000, table 5): nebular 
lightning, supernova shock waves, magnetic reconnection flares, gas dynamic (nebular) shock wave and planetesimal bow shocks. We also evaluate the following mechanisms, that were postulated since Rubin (2000): shocks in the envelopes of giant protoplanets (Nelson and Ruffert 2005) and current sheets in magnetically active nebula regions (Joung et al. 2004).

\subsubsection{Constraints from the coexistence with the matrix, and scrutinization of various heat sources}

The first line of evaluation considers the following five constraints. With them, we attempt to scrutinize the heating mechanisms.

a) The matrix materials are characterized by their very fine-grained nature, typically sub $\mu \mathrm{m}$ to $\mu \mathrm{m}$ in size. Such fine dust move with gas and are unable to be decoupled from the gas.

b) The matrix contains abundant materials and elements that are stable at low temperatures. Although recondensation or regeneration (if such processes followed the destruction during chondrule formation events) might partially restore their low-T features, most indigenous materials such as IOMs (e.g., Alexander et al. 2007) which are suggested as having inherited their isotopic signatures from the low-T, outer disk environments or from the molecular cloud would not survive even a moderate temperature rise.

c) The matrix contains presolar grains whose isotopic compositions (e.g., Zinner 1998) are very distinct from those of the common chondrite-forming minerals. Any event, thermal or shock, strong enough to destroy or alter the grains is prohibited.

d) The most stringent of all the constraints comes from the $\mathrm{D} / \mathrm{H}$ ratios of water and organic materials in matrices in various chondrites, which differ from those of the sun and the earth. As already described in Section 4.1.2, different chondrite types appear to possess characteristic ranges in the $\mathrm{D} / \mathrm{H}$ ratio, which are most likely attributed to the ion-molecule reactions in the molecular cloud (e.g., Tielens 2013) followed by modification in the low-T outer disk (e.g., Alexander et al. 2012; Cleeves et al. 2014). The $\mathrm{D} / \mathrm{H}$ ratio of water should be easily reset to that of the nebular gas, $\sim 2 \times 10^{-5}$, if temperature exceeded $500 \mathrm{~K}$ (Lécluse and Robert 1994) in the presence of the nebular gas.

e) Complementary nature of the matrix to the chondrules. Two kinds of complementarity may be identified. 1) Chondrules and matrix show similar isotopic compositions and redox states, as in the case of ordinary and enstatite chondrites. Carbonaceous chondrites, whose matrices are relatively $\mathrm{H}_{2} \mathrm{O}$-rich, possess oxidized matrices and reduced chondrules, but the oxidation of the matrices are known to have resulted from later hydrothermal actions after accumulation onto a large parent body (e.g., DuFresne and Anders 1962; Clayton and Mayeda 1984). 'Similarity' rather than complementarity may be a better term, but it indicates that both come from the same source, not a chance mixture of different origins. 2) Excess or deficit in abundances of particular elements or isotopes in either of chondrules or matrix cancels out to normal values when chondrules and matrix are combined in their chondritic proportion, viz., the bulk chondrite. It is recognized that chondrules are depleted in metallic elements, as opposed to enrichment in these elements in the matrix, while the bulk chondrite have approximately CI abundances in these elements (Bland et al. 2005). This suggests that the metallic elements lost from the chondrules were mostly recovered by the matrix (e.g., Grossman and Wasson 1985). Isotopic complementarity is a very severe restriction, especially in non-mass-dependent isotopic fractionations, as it suggests some sort of genetic link between them Budde et al. (2016a, b ).

The above four constraints, viz., a, b, c and d disqualify the heat sources or heating mechanisms which raise temperatures of the gas on a large scale in the disk, where fine dust, a source of matrix materials, are immersed. They include supernova and nebular shock waves, magnetic reconnection flares, current sheets, and shocks in the envelopes of giant protoplanets.

Planetesimal bow shock and lightning discharge should involve only localized heating of the disk gas, thus most of the fine dust in the disk would escape from heating. They are scrutinized by the second line of evaluation method in the followings.

\subsubsection{Scrutinization of planetesimal bow shock model}

As described in "Origin of Chondrule Chemistry" in paper (I) and in Section 3.5 in the present paper, jetdroplets are postulated to have ejected from the boiling surface of dustons starting with vaporization degree of MLB from less than $10 \%$ and ending with more than $80 \%$. In our experiments (paper I), the MLB completed evaporation (VD >90\%) within several seconds. A large size of duston would require more time, typically $\geq 10$ seconds for jet-droplets to be ejected with a wide range of $\mathrm{VD}$.

A high speed planetesimal moving in PPD (with a typical speed of $6 \mathrm{~km} \mathrm{~s}^{-1}$ relative to the gas) accompanies a bow shock on its front side. According to Ciesla et al. (2004), the thickness of bow shocked zone is about three times of the radius $\left(R_{p}\right)$ of the planetesimal. With $R_{p}=$ 10,100 or $1000 \mathrm{~km}$, its thickness is 30,300 or $3000 \mathrm{~km}$. 
Then, large dust aggregates immersed in the gas would be heated for duration of 5,50 or 500 seconds, which is long enough in our criteria. The calculated temperature of a compact dust aggregate of $0.1 \mathrm{~cm}$ in diameter in the bow shock, however, reaches only $1600 \mathrm{~K}$. Even with an assumed emissivity of dust being 0.1 , which is unrealistically low, the maximum temperature reached is $2000 \mathrm{~K}$, which is still too low to make the dust aggregate boil. Hood (1998) obtained a maximum temperature $2000 \mathrm{~K}$ for a dust aggregate by assuming a planetesimal of $R_{p}=70$ $\mathrm{km}$ and the same values for size and emissivity of dust grain as Ciesla et al. (2004).

Much larger dust aggregates like dustons $(>3 \mathrm{~cm})$ would decouple from the gas more efficiently and hence would be more strongly heated in bow shock, although we do not have a quantitative estimate. Even if boiling occurred, however, the vapor cloud would be immediately destroyed by shock wave. Dustons would be also smashed into pieces by impact with the planetesimal.

Miura et al. (2010) have proposed a possibility of liquid condensation for origin of chondrules that fine dust in the bow shock once evaporate and then condense. They, however, admitted that the condensed material should have the same composition as that of the former dust. Then it would not cause chemical fractionations in chondrules and chondrites from the dust.

We conclude that the bow shock is not a viable heat source for our model of chondrule formation.

\subsubsection{Lightning discharge: our last hope}

In the terrestrial atmosphere, lightning discharge releases its energy in an extremely confined path (a typical discharge path width is $\sim 2 \mathrm{~mm}$; Pilipp et al. 1992). This leaves most of the atmospheric volume free of heating except for vicinity of the path which are heated with radiation (Whipple 1966). The path width of lightning discharge postulated for PPD is $\sim 5000$ times of the electron mean free path, viz., $\sim 5 \mathrm{~km}$ for gas density $\mathrm{n}\left(\mathrm{H}_{2}\right)$ $\sim 10^{20} \mathrm{~m}^{-3}$ (Pilipp et al. 1992), which is very narrow considering the spatial scale of PPD. Therefore, the lightning discharge clears the first line of evaluation procedure, the coexistence of the matrix.

Muranushi (2010) has raised three problems to be overcome for the lightning discharge to be a feasible heat source for chondrule formation: energetics problem, neutralization problem, and destruction problem. We add another constraint: charging-mechanism problem. In the followings we give preliminary but plausible solutions to the four problems, and show that the lightning discharge is compatible with our model of chondrule and chondrite formation.

Charging mechanism The generally accepted concept for the development of the thundercloud charge dipole in the terrestrial atmosphere is the physical separation of oppositely charged particles within the cloud (Saunders 2008). The principal charge carriers are believed to be $\mathrm{H}_{2} \mathrm{O}$ ice particles and their charge separation mechanism has been studied for a long time (reviewed by National Research Council 1986). The $\mathrm{H}_{2} \mathrm{O}$ ice is the most abundant solid substance in PPD and is naturally assumed as a main charge carrier for lightning discharge in space.

We postulate that the chondrule formation occurred in large vortices in PPD as described in Section 4.2. Theoretical works predict that a pressure bump near the snow-line (e.g., Kretke and Lin 2007) induces Rossby wave instability by which anticyclonic vortices develop (e.g., Lovelace and Romanova 2014; Lin and Pierens 2018). If a large vortex (Huang et al. 2018) encompasses the snow-line, the icy dust trapped in it would experience evaporation and condensation in its every rotation period.

According to Saunders (2008), the most promising of all possible charging mechanisms of ice grains in the terrestrial atmosphere is a charging induced by condensation: If the growth rate of ice grains is fast during condensation, more defects (pairs of $\mathrm{OH}^{-}$and $\mathrm{H}_{3} \mathrm{O}^{+}$) are created on the ice surface. The $\mathrm{H}_{3} \mathrm{O}^{+}$diffuses into inside of the ice grains, leaving $\mathrm{OH}^{-}$trapped on the surface. When small ice particles collide with the large ice grains, they remove $\mathrm{OH}^{-}$ and become negatively charged, while the large ice grains having lost $\mathrm{OH}^{-}$become positively charged. Takahashi (1978) and Takahashi and Miyawaki (2002) conducted ice charging experiments showing that an ice crystal $(\sim 10$ $\mu \mathrm{m}$ in diameter) removed a maximum charge of $\sim 3.3 \times$ $10^{-14} \mathrm{C}$ per collision, which translates into a charge surface density of $10^{-4} \mathrm{C} \mathrm{m}^{-2}$. This is more than enough to explain activities of terrestrial thunderclouds (Takahashi 1978). According to Takahashi (1978), however, the charge surface density and its polarity depend strongly on temperature and density of $\mathrm{H}_{2} \mathrm{O}$ molecules in the atmosphere. Since there are no data available for the range of temperature and $\mathrm{H}_{2} \mathrm{O}$ density in PPD, it may be premature to conclude the charging efficiency per collision for nebular ice.

We find a similar situation in the vortex which by anticyclonic rotation would bring trapped dustons from warm (inside the snow-line) to cold (outside) environment. If the mass function of stony dust is biased to large sizes, viz., dustons and their embryos rather than fine dust, most of $\mathrm{H}_{2} \mathrm{O}$ vapor would not immediately condense but supersaturate up until homogeneous condensation generates a numerous number of ultra-fine ice particles (probably sub-micron in size). Dustons and their embryos, on the other hand, would be covered with an icy layer or frost. Dustons gradually decouple from the gas (which also contains fine dust of ice and stone) and spiral down into the center of the vortex, while the fine dust collide with the front and bottom sides of the dustons. 
They are similar to the large ice grains in the terrestrial atmosphere, that grow by condensation of water in updrafts of thunderclouds while small ice particles continuously collide with their bottoms. The separation of positively charged dustons and negatively charged ice particles develops an electric field between the two populations. Once the electric field is established, the dustons and their embryos are electrically polarized so that rebounding ice particles can deprive more negative charges from them, leaving them more positively charged. This results in an enforcement of the field (Saunders 2008). If the vortex radius is comparable to the scale height of the disk gas, it would look like a cylindrical capacitor with electric charges of opposite polarities arranged in concentric circles.

Energetics problem This problem has been recognized as a most formidable one since Whipple (1966) suggested a lightning discharge as a heat source of chondrules. The ultimate energy source (gravitational potential of the protoplanetary disk) is sufficient to melt the chondrules, but most of the energy earned by ingoing larger dust go to the outgoing gas by the angular momentum exchange; little contribute to the random motion, the energy source for the lightning (Muranushi 2010). According to our new chondrule formation mechanism, however, the energy shortage seems easy to overcome because only the surface, a few $\mathrm{mm}$ in thickness, of dustons needs melting and evaporation to generate chondrules. The larger the duston's average size, the smaller the total energy would be required if the total stony dust mass in the protoplanetary disk is assumed constant.

The energy source of lightning discharge in the terrestrial atmosphere is the latent heat of water vapor during its condensation in thunderclouds, partially transferred to turbulence and updraft in the developed cells, then partially to charge separation between icy grains (Rakov and Uman 2003). We assume the latent heat of water vapor as the source of the electric energy stored in the nebular vortex, and calculate the minimum radius of the average dustons, whose surface is exposed to lightning discharge and boils to produce jet-droplets and vapor cloud.

For simplicity, the mass ratio of ice to stone in the vortex is assumed to be $3: 1$, and all the stone is used for making dustons of single size (radius, $R$ ). It is assumed that all ice once evaporates on a warm side of the vortex and then condense totally on a cold side, releasing the latent heat. This latent heat is eventually used to heat the dustons. We further assume that the duston's surface with a thickness of $\Delta R$ is kept molten at $2600 \mathrm{~K}$, and that its one-third thickness, (1/3) $\Delta R$ evaporates. The initial temperature of the duston is assumed to be $100 \mathrm{~K}$. A thermal diffusion into the duston below the thickness $\Delta R$ is not considered. The adopted duston's thermodynamic properties are: $C_{p}=1.46 \mathrm{~kJ} \mathrm{~kg}^{-1} \mathrm{~K}^{-1} ; \Delta H_{m}=6.27 \times 10^{2} \mathrm{~kJ}$ $\mathrm{kg}^{-1} ; \Delta H_{v}=8.37 \times 10^{3} \mathrm{~kJ} \mathrm{~kg}^{-1}$ (all data refer to enstatite liquid; Chase et al. 1985). The water's heat of vaporization is $2.45 \times 10^{3} \mathrm{~kJ} \mathrm{~kg}^{-1}$. The radiative loss of energy (emissivity $=1$ ) from the duston surface is assumed equal to that for silicate vaporization, which is valid at $2600 \mathrm{~K}$ (Hashimoto and Nakano, in preparation).

We obtain $R / \Delta R \sim 4.0 \Phi_{c}^{-1}$, where $\Phi_{c}$ is the energy conversion efficiency of the water latent heat to the total energy involved in lightning discharge, multiplied by the conversion efficiency of the latter to the heating of dustons. If $\Delta R=3 \mathrm{~mm}$ and $\Phi_{c}=1, R=12 \mathrm{~mm}$. This would be a minimum size of dustons, but $\Phi_{c}$ would be significantly smaller than 1 ; if $\Phi_{c}=0.001$ (for the same $\Delta R=3 \mathrm{~mm}$ ), the duston's radius should be as large as $12 \mathrm{~m}$, which may be too large. Not all dustons would be struck by thunderbolts, but only their small proportion would be, considering the rarity and unpredictable nature of lightning on earth. This would reduce the optimum size of dustons significantly. If we presume $\Phi_{c}$ much smaller than 0.001 , the productivity of chondrules should be very low, but it would be more favorable from the energy limitation.

Neutralization problem The protoplanetary disk is not an ideal place for electric fields to build due to its low gas density. If the gas is even slightly ionized, the field is rapidly neutralized by Ohmic currents (Muranushi 2010). Because of the large mean free path of electrons in PPD compared to that in the earth atmosphere, the breakdown field is very small, of the order of $\sim 10 \mathrm{~V} \mathrm{~m}^{-1}$ (Muranushi et al. 2015), while on earth it is $\sim 10^{6} \mathrm{~V}$ $\mathrm{m}^{-1}$ (Rakov 2013). This prevents a large electric field from building up, as small discharges always wipe it out. Nevertheless, Pilipp et al. (1998) contended that global electric fields strong enough to induce lightning could have been generated by gravitational sedimentation of dust particles and size sorting due to gas drag on these particles (Morfill et al. 1993), (1) only if charge transfer processes operated in grain-grain collisions, (2) if the gas phase ionization rate were much lower than that usually assumed for molecular clouds, almost as low as that resulting from decay of long-lived radioactive elements $\left({ }^{40} \mathrm{~K}\right)$ trapped in the grains alone, (3) if precursors of chondrules were compact with a specific mass density not much lower than $1 \mathrm{~g} \mathrm{~cm}^{-3}$, (4) and if the electrical energy was stored in much larger structures. We agree to their assertions except that the driving force for charge separation is the Coriolis force in vortices rather than the disk gravity. The vertical component of gravity in the disk midplane, where much of dust are concentrated, is negligibly small; therefore an updraft with large velocities of unknown origin is needed to lift dust of various sizes to high altitudes, 
e.g., a disk scale height, where the vertical component becomes significant and causes separation of dust by size difference.

The vortices must have formed in an MRI-inactive, 'dead zone' (e.g., Kretke and Lin 2007), where the ionization source would be limited to radioactive elements and cosmic rays. Pilipp et al. (1998) assumed only ${ }^{40} \mathrm{~K}$ for ionization source, which they found marginal to keep a maximum allowable ionization from triggering global discharges. They did not consider a decay of ${ }^{26} \mathrm{Al}$, which could be a strong source of ionization. As Desch and Cuzzi (2000) pointed out, we may take it in a reversed logic that the chondrule formation occurred because ${ }^{26} \mathrm{Al}$ was already dead due to the time gap between chondrules and CAIs, or because ${ }^{26} \mathrm{Al}$ was not present in the chondrule formation zone due to its heterogeneous distribution (e.g., Larsen et al. 2011; Krot et al. 2012). We consent to the latter possibility because some types of chondrites (CO and LL chondrites) must have formed at the same time as CAIs, and another types of chondrites ( $\mathrm{CV}, \mathrm{CM}$ and $\mathrm{E}$ chondrites) could have formed even before the CAI formation. We also propose that any radioactive elements could not have been an effective ionization source because of the large size of dustons and because of the mass function biased to large aggregates. The electron (and perhaps positron) penetration depth is proportional to their energy, $\sim 1.7 \mathrm{~mm}$ per MeV (Kanaya and Okayama 1972). Therefore, only a few $\mathrm{mm}$ surface layer of the dustons may be liable to contribute to ionization of the gas; most of the mass which resides inside the dustons would not.

Destruction problem Muranushi (2010), in his rebuttal argument to the experiment by Güttler et al. (2008), pointed out that either the electron mean free path is many orders of magnitude shorter or the electron kinetic energy is much larger compared to the protoplanetary disk environment.

We rather pay attention to the conclusion by Güttler et al. (2008) that the fragmentation of experimental dust aggregates is attributed to the expansion of the discharge channel. In PPD the discharge channel is presumably very wide (e.g., $5 \mathrm{~km}$; Morfill et al. 1993) compared to that in the experiment (Güttler et al. 2008; no description but maybe $\leq 1 \mathrm{~mm}$ ) and the discharge would continue for much longer duration (e.g., 10-100 s; Morfill et al. 1993) as compared to $\sim 60 \mu \mathrm{s}$ in their experiment. Therefore, dust aggregates in the discharge channel in PPD would be completely molten before the channel explosively expands as a result of the vanishing magnetic fields around the channel.

\subsubsection{Lightning discharge: consistency with our new model} We place three conditions on evaluating the lightning discharge as a possible heating mechanism for chondrule formation: (i) the temperature of the duston's surface is kept above its boiling point for duration of $10 \mathrm{~s}$ or longer; (ii) the vapor cloud is kept for the same duration; and (iii) the matrix-forming fine dust coexist nearby at low temperatures. We also add one more condition, (iv) that the chondrules that formed in the lightning bolt should possess a large NRM (natural remnant magnetization) because of the magnetic field formed around the discharge path.

Morfill et al. (1993) estimated the energy available from a high temperature plasma of $\sim 30 \mathrm{eV}$ for heating dust grains in the discharge path, using a scaling of the typical terrestrial bolt of lightning applied to a low density nebular gas. Due to a large mean free path of electrons, $\sim 1$ $\mathrm{m}$ for $\mathrm{n}\left(\mathrm{H}_{2}\right) \sim 10^{20} \mathrm{~m}^{-3}$, the discharge path width is $\sim 5000 \mathrm{~m}$ and the duration of a lightning pulse is of the order 10-100 s (Morfill et al. 1993). Because of the wide cross section of the path and the prolonged duration of heating, the energy to impart to dust grains is diluted as opposed to the terrestrial one (width $\sim 1 \mathrm{~mm}$; duration $<1$ millisecond). This could be enough to melt grains as small as chondrules (Morfill et al. 1993; Horányi et al. 1995), but is in no way sufficient for boiling the surface of dustons.

We predict a new mechanism for heating dustons, a direct strike by the lightning bolt. The allowable electric field in the nebula is restricted by the dielectric strength of the molecular hydrogen. This limits the electric field inside vortices of the order of $10 \mathrm{~V} \mathrm{~m}^{-1}(0.14-30$ $\mathrm{V} \mathrm{m}^{-1}$ depending on the models of dielectric strength; Muranushi et al. (2015). This field is so weak compared to a typical terrestrial thundercloud field of $1-2 \times 10^{5} \mathrm{~V} \mathrm{~m}^{-1}$ (Rakov 2013) that a large scale charge separation, $10^{6}-10^{8}$ $\mathrm{m}$, is required to build an electric potential of $10^{7}-10^{9}$ $\mathrm{V}$, typical of terrestrial thunderclouds. Therefore, the discharge path in the nebular gas might be a very long trail. Our concern, however, is not the trail itself (which is usually assumed a heating scene of involved dust aggregates) but its destination: how the discharge tip of the stepped leader (e.g., Rakov and Uman 2003), a pathfinder so to speak, finds its partner in opposite polarity. Our hypothetical dustons are the one, since their 'point charge' fields stand out in the general field, like trees and lightning rods as a terrestrial analogue. One unlucky duston struck by the leader is among a large number of dustons which we postulate distributing equally-spaced in a plane that extends laterally more than thousands of $\mathrm{km}$. A swarm of secondary electrons and ions from the attacked duston may find another duston nearby, and the discharge process should cascade.

Once the duston's surface temperature rises high enough for evaporation of the icy layer and other volatiles, the first vapor cloud forms whose density exceeds that of the nebular gas by orders of magnitude. This would 
make a mean free path shorter by the same magnitude and converge the wide discharge path into a narrow string similar to the terrestrial lightning. From this on until discharge ends (for 10-100 s), the duston's surface continues to boil and the vapor cloud is maintained.

The cold fine dust exists outside of the discharge path. If the path is more confined by being shepherded by dustons, rather than being spread over kilometers, a nearby volume of the nebular gas around the vapor clouds may be also free of heating and ready to mix its contained fine dust with chondrules.

Wasilewski and Dickinson (2000) found that the tetrataenite-bearing chondrules in Bjurbole (L/LL4) and Chainpur (LL3) chondrites exhibited very high REM values (the ratio of NRM/SIRM) of $>100 \times 10^{-3}$, indicating that these chondrules were magnetized in a strong magnetic field of the order of $100 \mathrm{mT}(1000 \mathrm{G})$. According to their arguments, this high REM in the terrestrial rocks is associated only with a direct strike by lightning discharge. In our new chondrule formation mechanism, supercooled liquid droplets solidify and cool below the curie temperature $\left(770{ }^{\circ} \mathrm{C}\right.$ for tetrataenite; Herndon and Rowe 1974) inside the vapor cloud that exists only during the lightning discharge because the discharge path expands explosively at the moment electric currents vanish.

Our postulate, the lightning discharge as a heat source of boiling dustons, needs quantitative interrogation. We do not deny a more plausible mechanism to be found in future.

\section{Conclusions}

In paper (I), Nakano and Hashimoto have carried out evaporation and condensation experiments and applied the results to chondrules, explaining all the primary features of chondrules including their chemistry. On this basis they proposed a new model of chondrule formation that begins with dense and large aggregates of primordial CI-like dust, called 'dustons'. Jetdroplets are ejected from the boiling surface of duston and react with the vapor and the condensate while their traveling through the vapor cloud. Origin of chondrules.

This paper started as a sequel to paper (I), in that basically the same model and calculation procedure are applied to the bulk chemistry of chondrites, but having seen entirely new results on the origin of chondrites, we have expanded our view to the evolution of PPD materials to planetesimals. We highlight the following aspects in conclusions.

(i) The duston-vapor cloud model include the five components: jet-droplets, differential vapor, integrated vapor, final integrated vapor and $\mathrm{CI}^{*}$ dust. The first three components make chondrules and the last two components make the matrix of chondrites, altogether forming the bulk chondrite. CAIs which later mixed with the chondrite-forming components is added as a sixth and foreign component to the model to explain CAI-bearing chondrites. We have found that in four types of chondrites CAIs had mixed with the primordial dust before the chondrule formation. Ghost CAIs manifest themselves as unusual enrichment in refractory elements both in chondrules and in the bulk chondrite, and is added as a seventh component to the model. Seven types of chondrites, CV, CM, E, CO, LL, H and L, are applied with the numerical fitting procedures developed for reproduction of bulk chondrite compositions with parameters, VD (vaporization degree) and $f_{s}$ (redox indicator), to determine the compositions and molar proportions of the (maximum) seven components. We have obtained a single solution for each of the chondrite types.

(ii) The results of numerical fittings indicate that the average chondrule compositions of seven types of chondrites are achieved by significant evaporation with $24-43 \%$ in VD, and that the maximum evaporation experienced by the molten surface of dustons reach as large as $50-85 \%$ in VD. It not only proves that chondrules are not just melted drops of pre-existing dust aggregates, but most importantly vindicates that the chondrule formation is the driving force for the chemical fractionations in chondrites.

(iii) We have identified three groups of chondrites according to the parameter $f_{s}$ determined. The most reduced group, CV, CM and E, have $f_{s}=0.03-0.04$ and contain only CAIs; the middle group, $\mathrm{CO}$ and LL, have $f_{s}=0.20$ and contain both CAIs and ghost CAIs; and the most oxidized group, $\mathrm{H}$ and $\mathrm{L}$, have $f_{s}=0.22-0.27$ and contain a minor amount of ghost CAIs only. From these results we have derived the chronological sequence of chondrule formation for different types of chondrites: the chondrule formation for $\mathrm{CV}, \mathrm{CM}$ and $\mathrm{E}$ occurred before the invasion of CAI into the chondrule formation zone; it occurred during the CAI invasion for CO and LL; and it occurred after the CAI invasion for $\mathrm{H}$ and L. The concomitant increase in $f_{s}$ values with this chronological sequence indicates that the PPD environment changed from reducing to oxidizing in time. We have found that this sequence is also related to the $\mathrm{D} / \mathrm{H}$ ratios of various solar system and extrasolar substances. We propose that the $\mathrm{D} / \mathrm{H}$ ratio and the concentration of $\mathrm{O}_{2}$ molecules in PPD increased with time as PPD evolved. 
(iv) The chronological order of the chondrule formation and the CAI invasion revealed in this study is in conflict with the ${ }^{26} \mathrm{Al}$ chronology of CAIs and chondrules. We have found that dating of chondrules with live ${ }^{26} \mathrm{Al}$ is an artifact due to invasion of ghost CAIs before the chondrule formation, which contaminated the primordial dust with a live ${ }^{26} \mathrm{Al}$. The $\mathrm{Pb}-\mathrm{Pb}$ dating of CAIs and chondrules seems compatible with our result as long as CAIs and chondrules in the same chondrite are used.

(v) To complete the chemical fractionation, the chemically fractionated materials must be physically separated. Our model offers a natural selection according to the principle of aerodynamic friction in PPD because as a result of chondrule formation, materials are divided into three populations with different size ranges. The chondrule-cored, dust-mantled nodules and their clusters are supposed to be millimeters to centimeter in size; the dustons with heat-scorched surfaces are more than $3 \mathrm{~cm}$ in diameter; and the leftover fine condensates and indigenous primordial dust, that fail to stick to chondrules, would be typically less than $\mu \mathrm{m}$ in size.

(vi) We propose that the chondrule formation occurred inside the large vortices which encompass the snowline in PPD. We envisage that the dustons, whether heat-scorched or kept intact, fall faster into the center of the vortex and ultimately form a planetesimal while the chondrule-cored nodules fall slower and form a veneer on the planetesimal, thus completing a physical separation. The fine dust would not fall but slowly bail out from the vortex.

(vii) Having discussed our new ideas on the origin of the chemical fractionations in chondrites, we compare our model with those developed by Anders and his group, viz. the equilibrium condensation model. Our model provides a better explanation for the lithophile element fractionations in chondrites particularly because it explains the completion of the fractionation as a natural consequence of the chondrule formation from dustons, while the equilibrium condensation model has no clue to this problem. The condensation theory is in conflict with the metalsilicate fractionation observed in chondrites, while our model can be compatible because the fractionation must have occurred during or after the chondrule formation.

(viii) We have scrutinized a dozen heat sources for chondrule formation based on our new measures. Only one possible heat source is left compatible with our chondrule and chondrite formation model: lightning discharge. We propose that a large-scaled charge separation, 10-100 thousand kilometers apart inside the vortex, is possible between dustons and ice particles with opposite charges and induces direct discharge strikes on the surface of dustons for more than 10 seconds, energetic enough to make dustons boil and generate a swarm of chondrules. A direct strike is also compatible with the large NRM recorded in chondrules.

(ix) Chondrites are full of shocked records, which could be explained by their formation history that during the era of planet formation, violent planetesimalplanetesimal collisions should have caused their chondritic veneers stripped off. Fragments were later bound together with a weak self-gravity: rubble piled asteroids, which are likely sources of chondritic meteorites.

Finally, we have not positively included oxygen isotope data in the argument since our model, as it stands now, is not in need of those to construct it. A plethora of the isotopic data on chondrules, chondrites, CAIs, and other solar system materials, including Earth and Moon requires a full of consideration, which is difficult to do due to space limitation. Tactically, however, it will be wise to use the whole data not only for assessing our proposed model but also for guiding our coming works (Hashimoto et al., in preparation).

\section{APPENDIX}

\section{(A) Data}

All data in Table 1 are chemical compositions of silicates in $\mathrm{mol} \%$ on the ten element basis. The bulk chemical compositions of chondrites are mostly from the wetchemical analyses compiled by Jarosewich (1990). The wet-chemical method has several advantages compared to others, which are crucial in the present study. It usually uses a bulk of rock sample of 8-20 grams for each chondrite. Chondrites contain various petrographic units and mineral phases which are different in size, composition and chemical state. They are not necessarily homogeneously distributed inside the bulk chondrite, so that a few grams of rock sample may not represent its bulk composition. According to Jarosewich (1990), 20 grams of meteorite, whenever possible, is routinely allocated in order to assure homogeneous and representative powders. The wet-chemical analysis distinguishes between Feoxides, metals and sulfides. This is also necessary in our study.

The average composition of chondrules is a simple average of broad-beam EPMA data of individual chondrules that belong to a single chondrite or to a specified type of chondrites. Precision and reliability of such analyses are described in paper (I). Here the important fact is that those EPMA data represents only silicate portions of chondrules because metals and sulfides included in chondrules are routinely avoided in the analyses. 
Data sources of the bulk chondrite compositions and the average chondrule compositions, as well as adjustments (whenever necessary) to the data, are explained in the followings.

\section{Bulk chemical compositions}

1 L chondrite: The average composition of 54 L-chondrites (falls) in Jarosewich (1990, table 5), after normalization to $100 \mathrm{~mol} \%$ on the ten element basis, is shown.

$2 \mathrm{H}$ chondrite: The average composition of 26 H-chondrites (falls) in Jarosewich (1990, table 5), after normalization to $100 \mathrm{~mol} \%$ on the ten element basis, is shown.

3 LL chondrite: The average composition of 12 LL-chondrites (falls) in Jarosewich (1990, table 5), after normalization to $100 \mathrm{~mol} \%$ on the ten element basis, is shown.

4. CV chondrite: The bulk composition of Efremovka (CV3) in Jarosewich (1990, table 2), after normalization to $100 \mathrm{~mol} \%$ on the ten element basis, is shown. We choose Efremovka simply because Hezel and Palme (2010) have analyzed chondrules in it. Allende and Bali are also CV3 chondrites listed in Jarosewich (1990, table 2). There are many chondrule analyses available for Allende (see paper (I)). However, not only the matrix but also the chondrules in Allende are heavily altered, making it unsuitable for the present study.

5 E chondrite: The average composition of 10 E-chondrites, four in Jarosewich (1990, table 2) and six from Yanai et al. (1995) is used after some modification described below. In the wet-chemical analysis, usually the FeO content is obtained by subtraction of Fe metal and Fe sulfide from the total Fe content. This makes it difficult to determine the $\mathrm{FeO}$ content in good precision because the $\mathrm{FeO}$ content in $\mathrm{E}$ chondrites is very low by nature. In Jarosewich (1990, table 2), it is forced to zero because of this difficulty. According to Yanai et al. (1995), however, the least altered $\mathrm{E}$ chondrites contain 1-4 wt.\% FeO. Chondrules in the least altered Y-691 (E3) have their average FeO content 1.9 wt.\% (Ikeda 1983). Considering these data, we adopt 2 wt.\% for the bulk FeO content in E chondrites. The concentrations of other nine elements are simple averages of $10 \mathrm{E}$-chondrites. The total is normalized to $100 \mathrm{~mol} \%$ just as for other types of chondrites.

6 CO chondrite: The average bulk composition of 3 CO-chondrites in Jarosewich (1990, table 2), after normalization to $100 \mathrm{~mol} \%$ on the ten element basis, is shown. They are ALH-77003 (CO3), ALH-82101 (CO3) and Isna (CO3).
7 CM chondrite: The average bulk composition of 3 CM-chondrites in Jarosewich (1990, table 2), after normalization to $100 \mathrm{~mol} \%$ on the ten element basis, is shown. They are Banten (CM2), Y-791824 (CM2) and Y-793321 (CM2). Murchison (CM2) in Jarosewich (1990, table 2) is not included because of its unusually low Na content compared to others.

8 CI chondrite: The weighted average bulk composition of 3 CI-chondrites compiled by Lodders (2003, table 1 and 3) after normalization to $100 \mathrm{~mol} \%$ on the ten element basis, is shown. They are Orgueil (CI), Alais (CI) and Ivuna (CI).

$9 \mathrm{CI}^{*}$ : The composition after subtracting the half of $\mathrm{FeO}$ from the $\mathrm{CI}$ composition (explained in 8) and being renormalized to $100 \mathrm{~mol} \%$ is shown. See the text (Section 2.1) for more detail.

\section{Average composition of chondrules}

1 L chondrules: Average composition of 437 chondrules from three L-chondrites. They are Y-74191 (L3) (Ikeda and Takeda 1979; Kimura et al. 1979), ALH-77015 (L3) (Fujimaki et al. 1981; Nagahara 1981; Ikeda 1983) and ALH-77249 (L3) (Kimura 1983).

2 H chondrules: Average composition of 109 chondrules from two H-chondrites. They are ALH-77299 (H3.7) and Y-790986 (H3-4), both in Ikeda (1983). Ti and Mn data are transferred from those in the bulk $\mathrm{H}$ chondrite in Table 1 because the chondrule analyses frequently omitted these elements from the list of analysis.

3 LL chondrite: Average composition of 74 chondrules from ALH-764 (LL3) (Ikeda 1980). Ti and Mn data are transferred from those in the bulk LL chondrite in Table 1 because the chondrule analyses omitted these elements from the list of analysis.

4. CV chondrules: Average composition of 8 chondrules from Efremovka (CV3) (Hezel and Palme 2010).

5 E chondrules: Average composition of 88 chondrules from Y-691 (E3) (Ikeda 1983).

6 CO chondrules: Average composition of 110 chondrules from three CO-chondrites. They are ALH-77003 (CO3) (Ikeda 1982), Y-790992 (CO3) (Ikeda 1983) and Kainsaz (CO3) (Hezel and Palme 2010).

7 CM chondrules: Average composition of 10 chondrules from El-Quss Abu Said (CM) (Hezel and Palme 2010).

\section{(B) D/H Ratios}

The $\mathrm{D} / \mathrm{H}$ ratios of various solar system and extrasolar substances are shown in Fig. 10. Data and their references are shown in Table 6. 
Table 6 D/H ratios and references

\begin{tabular}{|c|c|c|c|}
\hline$\#$ & Material or Source & $\begin{array}{l}\text { D/H ratio } \\
\times 10^{-4}\end{array}$ & Reference \\
\hline 1 & Local interstellar medium (gas) & $0.15 \pm 0.01$ & Linsky (1998) \\
\hline 2 & ProtoSolar & $0.21 \pm 0.05$ & Geiss and Gloeckler (1998) \\
\hline 3 & Saturn (atmosphere) & $0.21 \pm 0.013$ & Pierel et al. (2017) \\
\hline 4 & Jupiter (atmosphere) & $0.295 \pm 0.055$ & Pierel et al. (2017) \\
\hline 5 & Uranus (atmosphere) & $0.44 \pm 0.04$ & Feuchtgruber et al. (2013) \\
\hline 6 & Neptune (atmosphere) & $0.41 \pm 0.04$ & Feuchtgruber et al. (2013) \\
\hline 7 & Tagish lake-11i & $0.164-1.14$ & Alexander et al. (2012) \\
\hline 8 & CV & $\leq 0.820$ & Alexander et al. (2012) \\
\hline 9 & $\mathrm{Cl}$ & $0.643-0.976$ & Alexander et al. (2012) \\
\hline 10 & $C M$ & $0.865 \pm 0.036$ & Alexander et al. (2012) \\
\hline 11 & $\mathrm{CO}$ & $0.849-1.32$ & Alexander et al. (2012) \\
\hline 12 & $C R$ & $1.71_{-0.1}^{+0.17}$ & Alexander et al. (2012) \\
\hline 13 & $\mathrm{O}$ & $2.8-3.44$ & Alexander et al. (2012) \\
\hline 14 & $\mathrm{R}$ & $7.26 \pm 0.13$ & McCanta et al. (2008) \\
\hline 15 & Earth Bulk & $1.49 \pm 0.03$ & Lécuyer et al. (1998) \\
\hline 16 & Comet 103P/Hartley 2 & $1.61 \pm 0.24$ & Hartogh et al. (2011) \\
\hline 17 & Comet 45P/Honda-Mrkos-Pajdušáková & $\leq 2$ & Lis et al. (2013) \\
\hline 18 & Comet 67P/Churymov-Gerasimenko & $5.3 \pm 0.7$ & Altwegg et al. (2015) \\
\hline 19 & Comet C/2009 P1 (Garradd) & $2.06 \pm 0.22$ & Bockelée-Morvan et al. (2012) \\
\hline 20 & Comet 153P/lkeya-Zhang & $\leq 2.5$ & Biver et al. (2006) \\
\hline 21 & Comet C/2002 T7 (Linear) & $2.5 \pm 0.7$ & Hutsemékers et al. (2008) \\
\hline 22 & Comet C/1996 B2 Hyakutake & $2.9 \pm 0.1$ & Bockelée-Morvan et al. (1998) \\
\hline 23 & Comet C/1995 O1 Hale-Bopp & $3.3 \pm 0.8$ & Meier et al. (1998) \\
\hline 24 & Comet P/Halley & $3.16 \pm 0.34$ & Eberhardt et al. (1995) \\
\hline 25 & Comet 8P/Tuttle & $4.09 \pm 1.45$ & Villanueva et al. (2009) \\
\hline 26 & NGC 1333 IRAS4B (Inner) & $5.9 \pm 1.7$ & Persson et al. (2014) \\
\hline 27 & NGC 1333 IRAS4B (Inner) & $1-37$ & Coutens et al. (2013) \\
\hline 28 & NGC 1333 IRAS4A (Inner) & 10 & Visser et al. (2013) \\
\hline 29 & NGC 1333 IRAS4A (Inner) & $4-30$ & Coutens et al. (2013) \\
\hline 29 & NGC 1333 IRAS4A (Outer) & $\approx 100$ & Coutens et al. (2013) \\
\hline 30 & NGC 1333 IRAS4A (Inner) & $19.1 \pm 5.4$ & Persson et al. (2014) \\
\hline 31 & NGC 1333 IRAS4A (Inner) & $50-300$ & Taquet et al. (2013) \\
\hline 32 & IRAS2A (Inner) & $7.4 \pm 2.1$ & Persson et al. (2014) \\
\hline 33 & IRAS2A (Inner) & $\geq 100$ & Liu et al. (2011) \\
\hline 33 & IRAS2A (Outer) & $900-1800$ & Liu et al. (2011) \\
\hline 34 & IRAS2A (Inner) & $30-800$ & Taquet et al. (2013) \\
\hline 35 & IRAS 16293-2422 (Inner) & $9.2 \pm 2.6$ & Persson et al. (2013) \\
\hline 36 & IRAS 16293-2422 (Inner) & 300 & Parise et al. (2005) \\
\hline 36 & IRAS 16293-2422 (Outer) & $\leq 20$ & Parise et al. (2005) \\
\hline 37 & IRAS 16293-2422 (Inner) & $140-580$ & Coutens et al. (2012) \\
\hline 37 & IRAS 16293-2422 (Outer) & $20-220$ & Coutens et al. (2012) \\
\hline
\end{tabular}

'Outer' and 'Inner' in parentheses denote outer and inner regions of clouds 


\section{Abbreviations}

VD: Vaporization degree; MLB: Mother liquid body; CROMs: C-rich refractory organic materials; JDMP: Jet-droplet mass percentage

\section{Acknowledgements}

We are greatly indebted to the members of the Planetary and Space Science group, Hokkaido University, for their valuable comments throughout this work and to Dr. A. Kouchi, Institute of Low Temperature Science, Hokkaido University for useful discussion. We thank the anonymous reviewers for their careful reading of our manuscript and their comments and suggestions.

\section{Authors' contributions}

$\mathrm{AH}$ and $\mathrm{YN}$ conceived the study. AH devised the numerical model. YN compiled the data base and surveyed astrophysical literature. AH and YN, together, developed the new model of chemical evolution of PPD materials. AH wrote the paper. All author(s) read and approved the final manuscript.

\section{Funding}

This research received no specific grant from any funding agency in the public, commercial, or not-for-profit sectors.

\section{Availability of data and materials}

The datasets supporting the conclusions of this article are included within the article and its appendices.

\section{Competing interests}

The authors declare that they have no competing interest.

\section{Author details}

${ }^{1}$ Suns \& Fishermen Society, Sapporo, Japan. ${ }^{2}$ Department of Cosmosciences, Hokkaido University, N10 W8, 060-0810, Sapporo, Japan.

\section{Received: 12 November 2019 Accepted: 21 October 2020} Published online: 22 January 2021

\section{References}

Alexander CMOD, Bowden R, Fogel ML, Howard KT, Herd CDK, Nittler LR (2012) The provenances of asteroids, and their contributions to the volatile inventories of the terrestrial planets. Science 337:721-723. https://doi.org/ 10.1126/science. 1223474

Alexander CMOD, Fogel M, Yabuta H, Cody GD (2007) The origin and evolution of chondrites recorded in the elemental and isotopic compositions of their macromolecular organic matter. Geochim Cosmochim Acta 71(17):4380-4403. https://doi.org/10.1016/j.gca.2007.06.052

Altwegg K, Balsiger H, Bar-Nun A, Berthelier JJ, Bieler A, Bochsler P, Briois C, Calmonte U, Combi M, De Keyser J, Eberhardt P, Fiethe B, Fuselier S, Gasc S, Gombosi TI, Hansen KC, Hässig M, Jäckel A, Kopp E, Korth A, LeRoy L, Mall U, Marty B, Mousis O, Neefs E, Owen T, Rëme H, Rubin M, Sémon T, Tzou C-Y, Waite H, Wurz P (2015) 67P/Churyumov-Gerasimenko, a jupiter family comet with a high D/H ratio. Science 347:1261952. https://doi.org/10. 1126/science.1261952

Anders E (1971) Meteorites and the early solar system. Annu Rev Astron Astrophys 9:1-34. https://doi.org/10.1146/annurev.aa.09.090171.000245

Bardyn A, Baklouti D, Cottin H, Fray N, Briois C, Paquette J, Stenzel O, Engrand C, Fischer H, Hornung K, Isnard R, Langevin Y, Lehto H, Roy LL, Ligier N, Merouane S, Modica P, Orthous-Daunay FR, Rynö J, Schulz R, Silén J, Thirkell L, Varmuza K, Zaprudin B, Kissel J, Hilchenbach M (2017) Carbon-rich dust in comet 67P/Churyumov-Gerasimenko measured by COSIMA/Rosetta. Mon Not R Astron Soc 469(Suppl_2):S712-S722. https:// doi.org/10.1093/mnras/stx2640

Barge P, Sommeria J (1995) Did planet formation begin inside persistent gaseous vortices? Astron Astrophys 295:L1-L4. https://ui.adsabs.harvard. edu/abs/1995A\%26A...295L...1B/abstract

Beitz E, Güttler C, Weidling R, Blum J (2012) Free collisions in a microgravity many-particle experiment - II: The collision dynamics of dust-coated chondrules. Icarus 218:701-706. https://doi.org/10.1016/j.icarus.2011.11.036

Bieler A, Altwegg K, Balsiger H, Bar-Nun A, Berthelier J-J, Bochsler P, Briois C, Calmonte U, Combi M, De Keyser J, van Dishoeck EF, Fiethe B, Fuselier SA, Gasc S, Gombosi TI, Hansen KC, Hässig M, Jäckel A, Kopp E, Korth A, Le Roy L, Mall U, Maggiolo R, Marty B, Mousis O, Owen T, Réme H, Rubin M, Sémon T, Tzou C-Y, Waite JH, Walsh C, Wurz P (2015) Abundant molecular oxygen in the coma of comet 67P/Churyumov-Gerasimenko. Nature 526:678-681. https://doi.org/10.1038/nature15707

Bischoff A, Keil K, Stöffler D (1984) Perovskite-hibonite-spinel-bearing, refractory inclusions and $\mathrm{Ca}$-Al-rich chondrules in enstatite chondrites. Geochemistry 44:97-106. https://ui.adsabs.harvard.edu/\#abs/1984Metic. 19..193B/abstract

Biver N, Bockelée-Morvan D, Crovisier J, Lis DC, Moreno R, Colom P, Henry F, Herpin F, Paubert G, Womack M (2006) Radio wavelength molecular observations of comets C/1999 T1 (McNaught-Hartley), C/2001 A2 (LINEAR), C/2000 WM1 (LINEAR) and 153P//keya-Zhang. Astron Astrophys 449:1255-1270. https://doi.org/10.1051/0004-6361:20053849

Bland PA, Alard O, Benedix GK, Kearsley AT, Menzies ON, Watt LE, Rogers NW (2005) Volatile fractionation in the early solar system and chondrule/matrix complementarity. PNAS 102:13755-13760. https://doi.org/10.1073/pnas. 0501885102

Bockelée-Morvan D, Biver N, Swinyard B, de Val-Borro M, Crovisier J, Hartogh P, Lis DC, Moreno R, Szutowicz S, Lellouch E, Emprechtinger M, Blake GA, Courtin R, Jarchow C, Kidger M, Küppers M, Rengel M, Davis GR, Fulton T, Naylor D, Sidher S, Walker H (2012) Herschel measurements of the D/H and ${ }^{16} \mathrm{O} /{ }^{18} \mathrm{O}$ ratios in water in the Oort-cloud comet C/2009 P1 (Garradd). Astron Astrophys 544:L15. https://doi.org/10.1051/0004-6361/201219744

Bockelée-Morvan D, Gautiera D, Lis DC, Young K, Keene J, Phillips T, Owen T, Crovisiera J, Goldsmit PF, Bergin EA, Despois D, Wootten A (1998) Deuterated water in comet C/1996 B2 (Hyakutake) and its implications for the origin of comets. Icarus 133:147-162. https://doi.org/10.1006/icar. 1998.5916

Bollard J, Connelly JN, Whitehouse MJ, Pringle EA, Bonal L, Jørgensen JK, Nordlund Å,, Moynier F, Bizzarro M (2017) Early formation of planetary building blocks inferred from Pb isotopic ages of chondrules. Sci Adv 3:e1700407. https://doi.org/10.1126/sciadv.1700407

Budde G, Burkhardt C, Brennecka GA, Fischer-Gödde M, Kruijer TS, Kleine T (2016a) Molybdenum isotopic evidence for the origin of chondrules and a distinct genetic heritage of carbonaceous and non-carbonaceous meteorites. Earth Planet Sci Lett 454:293-303. https://doi.org/10.1016/j. epsl.2016.09.020

Budde G, Kleine T, Kruijer TS, Burkhardt C, Metzler K (2016b) Tungsten isotopic constraints on the age and origin of chondrules. PNAS 113:2886-2891. https://doi.org/10.1073/pnas.1524980113

Cameron AGW (1962) The formation of the sun and planets. Icarus 1:13-69. https://doi.org/10.1016/0019-1035(62)90005-2

Cameron AGW (1963) Formation of the solar nebula. Icarus 1:339-342. https:// doi.org/10.1016/0019-1035(62)90033-7

Chase MW, Davies CA, Downey, Jr. JR, Frurip DJ, McDonald RA, Syverud AN (1985) Phys. Chem. Ref. Data. Suppl. No. 1. In: Chase MW, Davies CA, Downey, Jr. JR, Frurip DJ, McDonald RA, Syverud AN (eds). NIST-JANAF Thermochemical Tables, 3rd edn. American Chemical Society, Washington

Chavanis PH (2000) Trapping of dust by coherent vortices in the solar nebula. Astron Astrophys 356:1089-1111. https://ui.adsabs.harvard.edu/\#abs/ 2000A\&A...356.1089C/abstract

Ciesla FJ, Hood LL, Weidenschilling SJ (2004) Evaluating planetesimal bow shocks as sites for chondrule formation. Meteorit Planet Sci 39:1809-1821. https://doi.org/10.1111/j.1945-5100.2004.tb00077.x

Clayton RN, Mayeda TK (1977) Correlated oxygen and magnesium isotope anomalies in Allende Inclusions, I: Oxygen. Geophys Res Lett 4:295-298. https://doi.org/10.1029/GL004i007p00295

Clayton RN, Mayeda TK (1984) The oxygen isotope record in Murchison and other carbonaceous chondrites. Earth Planet Sci Lett 67:151-161. https:// doi.org/10.1016/0012-821X(84)90110-9

Cleeves LI, Bergin EA, Alexander CMOD, Du F, Graninger D, Öberg KI, Harries TJ (2014) The ancient heritage of water ice in the solar system. Science 345:1590-1593. https://doi.org/10.1126/science.1258055

Codella C, Ceccarelli C, Cabrit S, Gueth F, Podio L, Bachiller R, Fontani F, Gusdorf A, Lefloch B, Tafalla M (2016) Water and acetaldehyde in HH212: The first hot corino in Orion. Astron Astrophys 586:L3. https://doi.org/10. 1051/0004-6361/201527424

Connelly JN, Bizzarro M, Krot AN, Nordlund Å, Wielandt D, Ivanova MA (2012) The absolute chronology and thermal processing of solids in the solar protoplanetary disk. Science 338:651-655. https://doi.org/10.1126/science. 1226919

Coutens A, Vastel C, Cabrit S, Codella C, Kristensen LE, Ceccarelli C, van Dishoeck EF, Boogert ACA, Bottinelli S, Castets A, Caux E, Comito C, Demyk 
K, Herpin F, Lefloch B, McCoey C, Mottram JC, Parise B, Taquet V, van der Tak FFS, Visser R, Yildiz UA (2013) Deuterated water in the solar-type protostars NGC 1333 IRAS 4A and IRAS 4B. Astron Astrophys 560:A39. https://doi.org/10.1051/0004-6361/201322400

Coutens A, Vastel C, Caux E, Ceccarelli C, Bottinelli S, Wiesenfeld L, Faure A, Scribano Y, Kahane C (2012) A study of deuterated water in the low-mass protostar IRAS 16293-2422. Astron Astrophys 539:A132. https://doi.org/10. 1051/0004-6361/201117627

Davis AM, McKeegan KD (2014) Short-lived radionuclides and early solar system chronology. In: Davis AM (ed). Treatise on Geochemistry, Vol.1, Meteorites and Cosmochemical Processes, 2nd Edition. Elsevier-Pergamon, Oxford. pp 361-395. https://www.sciencedirect.com/referencework/ 9780080983004/treatise-on-geochemistry\#browse-content

Deloule E, Robert F (1995) Interstellar water in meteorites? Geochim Cosmochim 59:4695-4706. https://doi.org/10.1016/0016-7037(95)00313-4

Desch SJ, Cuzzi JN (2000) The generation of lightning in the solar nebula. Icarus 143:87-105. https://doi.org/10.1006/icar.1999.6245

Dobricą E, Engrand C, Leroux H, Rouzaud J-N, Duprata J (2012) Transmission electron microscopy of CONCORDIA UltraCarbonaceous Antarctic MicroMeteorites (UCAMMs): Mineralogical properties. Geochim Cosmochim 76:68-82. https://doi.org/10.1016/j.gca.2011.10.025

Dufresne ER, Anders E (1962) On the chemical evolution of the carbonaceous chondrites. Geochim Cosmochim 26:1085-1092. https://doi.org/10.1016/ 0016-7037(62)90047-9

Dullemond CP, Hollenbach D, Kamp I, D'Alessio P (2007) Models of the structure and evolution of protoplanetary disks. In: Reipurth B, Jewitt D, Keil K (eds). Protostars and Planets V. The University of Arizona Press, Tucson. pp 555-572. https://www.lpi.usra.edu/books/PPV/download.html

Eberhardt P, Reber M, Krankowsky D, Hodges RR (1995) The D/H and ${ }^{18} \mathrm{O} /{ }^{16} \mathrm{O}$ ratios in water from comet P/Halley. Astron Astrophys 302:301-316. http:// adsabs.harvard.edu/abs/1995A\%26A...302..301E

Eistrup C, Walsh C (2019) Formation of cometary $\mathrm{O}_{2}$ ice and related ice species on grain surfaces in the midplane of the pre-solar nebula. Astron Astrophys 621:A75. https://doi.org/10.1051/0004-6361/201833380

Engrand C, Duprat J, Dartois E, Benzerara K, Leroux H, Baklouti D, Bardyn A, Briois C, Cottin H, Fischer H, Fray N, Godard M, Hilchenbach M, Langevin Y, Paquette J, Rynö J, Schulz R, Silén J, Stenzel O, Thirkell L, the COSIMA team (2016) Variations in cometary dust composition from Giotto to Rosetta, clues to their formation mechanisms. Mon Not R Astron Soc 462:S323-S330. https://doi.org/10.1093/mnras/stw2844

Fagan TJ, Krot AN, Keil K (2000) Calciumaluminumrich inclusions in enstatite chondrites (I): Mineralogy and textures. Meteorit Planet Sci 35:771-781. https://doi.org/10.1111/j.1945-5100.2000.tb01461.x

Feuchtgruber H, Lellouch E, Orton G, de Graauw T, Vandenbussche B, Swinyard B, Moreno R, Jarchow C, Billebaud F, Cavalié T, Sidher S, Hartogh $P$ (2013) The D/H ratio in the atmospheres of Uranus and Neptune from Herschel-PACS observations. Astron Astrophys 551:A126. https://doi.org/ 10.1051/0004-6361/201220857

Friedrich JM, Perrotta GC, Kimura M (2014) Compositions, geochemistry, and shock histories of recrystallized LL chondrites. Geochim Cosmochim Acta 139:83-97. https://doi.org/10.1016/j.gca.2014.04.044

Fromang S, Nelson RP (2005) On the accumulation of solid bodies in global turbulent protoplanetary disc models. Mon Not R Astron Soc 364:L81-L85. https://doi.org/10.1111/j.1745-3933.2005.00109.x

Fujimaki H, Matsu-ura M, Sunagawa I, Aoki K (1981) Chemical compositions of chondrules and matrices in the ALH-77015 chondrite (L3). Memoirs Natl Inst Polar Res 20:161-174. https://nipr.repo.nii.ac.jp/?action=pages_view_ main\&active_action=repository_view_main_item_detail\&item_id $=1211$ \& item_no=1\&page_id=13\&block_id=104. Special issue

Furuya K, Aikawa Y, Nomura H, Hersant F, Wakelam V (2013) Water in protoplanetary disks: Deuteration and turbulent mixing. Astrophys $J$ 779(1):11. https://doi.org/10.1088/0004-637X/779/1/11

Geiss J, Gloeckler G (1998) Abundances of deuterium and helium-3 in the protosolar cloud. Space Sci Rev 84:239-250. https://doi.org/10.1023/A: 1005039822524

Grossman JN (1972) Condensation in the primitive solar nebula. Geochim Cosmochim Acta 36:597-619. https://doi.org/10.1016/00167037(72)90078-6

Grossman JN, Steele IM (1976) Amoeboid olivine aggregates in the Allende meteorite. Geochim Cosmochim Acta 40:149-150. https://doi.org/10. 1016/0016-7037(76)90172-1
Grossman JN, Wasson JT (1985) The origin and history of the metal and sulfide components of chondrules. Geochim Cosmochim Acta 49:925-939. https://doi.org/10.1016/0016-7037(85)90308-4

Guan Y, Huss GR, MacPherson GJ, Wasserburg GJ (2000) Calcium-aluminumrich inclusions from enstatite chondrites: Indigenous or foreign? Science 289:1330-1333. https://doi.org/10.1126/science.289.5483.1330

Güttler C, Poppe T, Wasson JT, Blum J (2008) Exposing metal and silicate charges to electrical discharges: Did chondrules form by nebular lightning? Icarus 195:504-510. https://doi.org/10.1016/j.icarus.2007.11.021

Hartmann L, Herczeg G, Calvet N (2016) Accretion onto pre-main-sequence stars. Annu Rev Astron Astrophys 54:135-180. https://doi.org/10.1146/ annurev-astro-081915-023347

Hartogh P, Lis DC, Bockelée-Morvan D, de Val-Borro M, Biver N, Küppers M, Emprechtinger M, Bergin EA, Crovisier J, Rengel M, Moreno R, Szutowicz S, Blake GA (2011) Ocean-like water in the Jupiter-family comet 103P/Hartley 2. Nature 478:218-220. https://doi.org/10.1038/nature10519

Hashimoto A, Grossman L (1987) Alteration of Al-rich inclusions inside amoeboid olivine aggregates in the Allende meteorite. Geochim Cosmochim Acta 51:1685-1704. https://doi.org/10.1016/00167037(87)90348-6

Herndon JM, Rowe MW (1974) Magnetism in meteorites. Meteoritics 9:289-305. https://doi.org/10.1111/j.1945-5100.1974.tb01197.x

Hewins RH, Zanda B, Bendersky C (2012) Evaporation and recondensation of sodium in Semarkona type II chondrules. Geochim Cosmochim Acta 78:1-17. https://doi.org/10.1016/j.gca.2011.11.027

Hezel DC, Palme H (2010) The chemical relationship between chondrules and matrix and the chondrule matrix complementarity. Earth Planet Sci Lett 294:85-93. https://doi.org/10.1016/j.epsl.2010.03.008

Hiyagon $\mathrm{H}$, Hashimoto A (1999) ${ }^{16} \mathrm{O}$ excesses in olivine inclusions in Yamato-86009 and Murchison chondrites and their relation to CAls. Science 283:828-831. https://doi.org/10.1126/science.283.5403.828

Hood LL (1998) Thermal processing of chondrule precursors in planetesimal bow shocks. Meteorit Planet Sci 33:97-107. https://doi.org/10.1111/j.19455100.1998.tb01611.x

Horányi M, Morfill G, Goertz CK, Levy EH (1995) Chondrule Formation in Lightning Discharges. Icarus 114:174-185. https://doi.org/10.1006/icar. 1995.1052

Huang P, Isella A, Li H, Li S, Ji J (2018) Identifying anticyclonic vortex features produced by the Rossby wave instability in protoplanetary disks. Astrophys J 867(1):3. https://doi.org/10.3847/1538-4357/aae317

Hutcheon ID, Hutchison R (1989) Evidence from the Semarkona ordinary chondrite for ${ }^{26} \mathrm{~A} 1$ heating of small planets. Nature 337:238-241. https:// doi.org/10.1038/337238a0

Hutsemékers D, Manfroid J, Jehin E, Zucconi J-M, Arpigny C (2008) The ${ }^{16} \mathrm{OH} /{ }^{18} \mathrm{OH}$ and $\mathrm{OD} / \mathrm{OH}$ isotope ratios in comet $\mathrm{C} / 2002 \mathrm{~T} 7$ (LINEAR). Astron Astrophys 490:L31-L34. https://doi.org/10.1051/0004-6361:200810833

Ikeda Y (1980) Petrology of Allan hills-764 Chondrites (LL3). Memoirs Natl Inst Polar Res 17:50-82. https://nipr.repo.nii.ac.jp/?action=pages_view_main\& active_action=repository_view_main_item_detail\&item_id=1089\&item_ no=1\&page_id=13\&block_id=104. Special issue

Ikeda Y (1982) Petrology of the ALH-77003 Chondrite (C3). Memoirs Natl Inst Polar Res 25:34-65. https://nipr.repo.nii.ac.jp/?action=pages_view_main\& active_action=repository_view_main_item_detail\&item_id=1378\&item no $=1 \&$ page_id $=13 \&$ block_id $=104$. Special issue

Ikeda Y (1983) Alteration of chondrules and matrices in the four antarctic carbonaceous chondrites ALH-77307 (C3), Y-790123 (C2), Y-75293 (C2), and Y-74662 (C2). Memoirs Natl Inst Polar Res 30:93-108. https://nipr.repo. nii.ac.jp/?action=pages_view_main\&active_action=repository_view_ main_item_detail\&item_id $=1554 \&$ item_no $=1$ \&page_id $=13 \& b$ lock_id $=$ 104. Special issue

Ikeda Y, Takeda H (1979) Petrology of the Yamato-74191 Chondrite. Memoirs Natl Inst Polar Res 12:38-58. https://nipr.repo.nii.ac.jp/?action=pages view_main\&active_action=repository_view_main_item_detail\&item_id= 958\&item_no=1\&page_id=13\&block_id=104. Special issue

Ireland TR (1988) Correlated morphological, chemical, and isotopic characteristics of hibonites from the Murchison carbonaceous chondrite. Geochim Cosmochim Acta 52:2877-2839. https://doi.org/10.1016/00167037(88)90150-0

Jacquet $E$ (2014) The quasi-universality of chondrule size as a constraint for chondrule formation models. Icarus 232:176-186. https://doi.org/10.1016/ j.icarus.2014.01.012 
Jarosewich E (1990) Chemical analyses of meteorites: A compilation of stony and iron meteorite analyses. Meteoritics 25:323-337. https://doi.org/10. 1111/j.1945-5100.1990.tb00717.x

Jones RH (1990) Petrology and mineralogy of type II, FeO-rich chondrules in Semarkona (LL3.0): Origin by closed-system fractional crystallization, with evidence for supercooling. Geochim Cosmochim Acta 54:1785-1802. https://doi.org/10.1016/0016-7037(90)90408-D

Jones RH (1996) FeO-rich, porphyritic pyroxene chondrules in unequilibrated ordinary chondrites. Geochim Cosmochim Acta 60:3115-3117. https://doi. org/10.1016/0016-7037(96)00152-4

Joung MKR, Mac Low MM, Ebel DS (2004) Chondrule formation and protoplanetary disk heating by current sheets in nonideal magnetohydrodynamic turbulence. Astrophys J 606:532-541. https://doi. org/10.1086/381651

Jørgensen JK, van Dishoeck EF (2010) The $\mathrm{HDO} / \mathrm{H}_{2} \mathrm{O}$ ratio in gas in the inner regions of a low-mass protostar. Astrophys J Lett 725:L172-L175. https:// doi.org/10.1088/2041-8205/710/1/L72

Kanaya K, Okayama S (1972) Penetration and energy-loss theory of electrons in solid targets. J Phys D Appl Phys 5:43-58. https://doi.org/10.1088/0022$3727 / 5 / 1 / 308$

Keays R, Ganapathy R, Anders E (1971) Chemical fractionations in meteorites-IV abundances of fourteen trace elements in L-chondrites; implications for cosmothermometry. Geochim Cosmochim Acta 35:337-368. https://doi.org/10.1016/0016-7037(71)90078-0

Keller LP, Messenger S (2011) On the origins of GEMS grains. Geochim Cosmochim Acta 75:5336-5365. https://doi.org/10.1016/j.gca.2011.06.040

Kimura M (1983) Chemical and petrologic relations of the constituent units in ALH-77249 meteorite (L3). Memoirs Natl Inst Polar Res 30:146-167. https:// nipr.repo.nii.ac.jp/?action=pages_view_main\&active_action=repository_ view_main_item_detail\&item_id=1557\&item_no=1\&page_id=13\&block_ $\mathrm{id}=104$. Special issue

Kimura M, Yagi K, Onuma K (1979) Petrological Studies on Chondrules in Yamato-74 Meteorites. Memoirs Natl Inst Polar Res 12:114-133. https:// nipr.repo.nii.ac.jp/?action=pages_view_main\&active_action=repository view_main_item_detail\&item_id=963\&item_no=1\&page_id=13\&block_ $\mathrm{id}=104$. Special issue

Kita NT, Nagahara H, Togashi S, Morishita Y (2000) A short duration of chondrule formation in the solar nebula: evidence from ${ }^{26} \mathrm{Al}$ in Semarkona ferromagnesian chondrules. Geochim Cosmochim Acta 64:3913-3922. https://doi.org/10.1016/S0016-7037(00)00488-9

Kita NT, Ushikubo T (2012) Evolution of protoplanetary disk inferred from ${ }^{26} \mathrm{Al}$ chronology of individual chondrules. Meteorit Planet Sci 47:1108-1119. https://doi.org/10.1111/j.1945-5100.2011.01264.x

Kothe S, Güttler C, Blum J (2010) The physics of protoplanetesimal dust agglomerates. $\vee$, Multiple impacts of dusty agglomerates at velocities above the fragmentation threshold. Astrophys J 725:1242-1251. https:// doi.org/10.1088/0004-637X/725/1/1242

Kretke KA, Lin DNC (2007) Grain retention and formation of planetesimals near the snow line in MRI-driven turbulent protoplanetary disks. Astrophys J 664:L55-L58. https://doi.org/10.1086/520718

Krot AN, Makide K, Nagashima K, Huss GR, Ogliore RC, Ciesla FJ, Yang L, Hellebrand E, Gaidos E (2012) Heterogeneous distribution of ${ }^{26} \mathrm{Al}$ at the birth of the solar system: Evidence from refractory grains and inclusions. Meteorit Planet Sci 47:1984-1979. https://doi.org/10.1111/maps.12008

Krot AN, Petaev MI, Scott ERD, Choi B-G, Zolensky ME, Keil K (1998) Progressive alteration in CV3 chondrites: More evidence for asteroidal alteration. Meteorit Planet Sci 33:1065-1085. https://doi.org/10.1111/j.1945-5100. 1998.tb01713.x

Kunihiro T, Rubin AE, McKeegan KD, Wasson JT (2004) Initial ${ }^{26} \mathrm{Al} /{ }^{27} \mathrm{Al}$ in carbonaceous-chondrite chondrules: too little ${ }^{26} \mathrm{Al}$ to melt asteroids. Geochim Cosmochim Acta 68:2947-2957. https://doi.org/10.1016/j.gca. 2004.02.006

Kurahashi E, Kita NT, Nagahara H, Morishita Y (2008) ${ }^{26} \mathrm{Al}-{ }^{26} \mathrm{Mg}$ systematics of chondrules in a primitive CO chondrite. Geochim Cosmochim Acta 72:3865-3882. https://doi.org/10.1016/j.gca.2008.05.038

Larimer JW (1979) The condensation and fractionation of refractory lithophile elements. Icarus 40:446-454. https://doi.org/10.1016/00191035(79)90038-1

Larimer JW, Anders E (1970) Chemical fractionations in meteorites-III, Major element fractionations in chondrites. Geochim Cosmochim Acta 34:367-387. https://doi.org/10.1016/0016-7037(70)90112-2
Larsen KK, Trinquier A, Paton C, Schiller M, Wielandt D, Ivanova MA, Connelly JN, Nordlund Å, Krot AN, Bizzarro M (2011) Evidence for magnesium isotope heterogeneity in the solar protoplanetary disk. Astrophys J Lett 735:L37. https://doi.org/10.1088/2041-8205/735/2/L37

Laul JC, Ganapathy R, Anders E, Morgan JW (1973) Chemical fractionations in meteorites-VI, Accretion temperatures of $\mathrm{H}$-, LL- and E-chondrites, from abundance of volatile trace elements. Geochim Cosmochim Acta 37:329-357. https://doi.org/10.1016/0016-7037(73)90138-5

Lécluse C, Robert F (1994) Hydrogen isotope exchange reaction rates: Origin of water in the inner solar system. Geochim Cosmochim Acta 58:2927-2939. https://doi.org/10.1016/0016-7037(94)90126-0

Lécuyer C, Gillet P, Robert F (1998) The hydrogen isotope composition of seawater and the global water cycle. Chem Geol 145:249-261. https://doi. org/10.1016/S0009-2541(97)00146-0

Lin M-K, Pierens A (2018) Vortex survival in 3D self-gravitating accretion discs. Mon Not R Astron Soc 478:575-591. https://doi.org/10.1093/mnras/sty947

Linsky JL (1998) Deuterium abundance in the local ism and possible spatial variations. Space Sci Rev 84:285-296. https://doi.org/10.1023/A: 1005048124341

Lis DC, Biver N, Bockelée-Morvan D, Hartogh P, Bergin EA, Blake GA, Crovisier J, de Val-Borro M, Jehin E, Küppers M (2013) A HERSCHEL study of D/H in water in the jupiter-family comet 45P/Honda-Mrkos-Pajdušáková and prospects for D/H measurements with CCAT. Astrophys J Lett 774:L3. https://doi.org/10.1088/2041-8205/774/1/L3

Liu F-C, Parise B, Kristensen L, Visser R, van Dishoeck EF, Güsten R (2011) Water deuterium fractionation in the low-mass protostar NGC1333-IRAS2A. Astron Astrophys 527:A19. https://doi.org/10.1051/0004-6361/201015519

Lodders K (2003) Solar system abundances and condensation temperatures of the elements. Astrophys J 591:1220-1247. https://doi.org/10.1086/375492

Lodders K, Palme H, Gail HP (2009) Abundances of the elements in the solar system. In: Trümper JE (ed). Landolt-Börnstein, New Series, Astronomy and Astrophysics. Springer, Berlin. pp 560-630

Lovelace RVE, Romanova MM (2014) Rossby wave instability in astrophysical discs. Fluid Dyn Res 46(4):041401. https://doi.org/10.1088/0169-5983/46/ 4/041401

MacPherson GJ (2014) Calcium-Aluminum-Rich Inclusions in Chondritic Meteorites. In: Davis AM (ed). Treatise on Geochemistry, Vol.1, Meteorites and Cosmochemical Processes, 2nd Edition. Elsevier-Pergamon, Oxford. pp 139-179. https://www.sciencedirect.com/referencework/ 9780080983004/treatise-on-geochemistry\#browse-content

MacPherson GJ, Bar-Matthew M, Tanaka T, Olsen E, Grossman L (1983) Refractory inclusions in the Murchison meteorite. Geochim Cosmochim Acta 47:823-839. https://doi.org/10.1016/0016-7037(83)90116-3

MacPherson GJ, Davis AM, Zinner EK (1995) The distribution of aluminum26 in the early Solar System-A reappraisal. Meteoritics 30:365-386. https://doi. org/10.1111/j.1945-5100.1995.tb01141.x

McCanta MC, Treiman AH, Dyar MD, Alexander CMOD, Rumble III D, Essene EJ (2008) The LaPaz Icefield 04840 meteorite: Mineralogy, metamorphism, and origin of an amphibole- and biotite-bearing R chondrite. Geochim Cosmochim Acta 72:5757-5780. https://doi.org/10.1016/j.gca.2008.07.034

Meier R, Owen TC, Matthews HE, Jewitt DC, Bockelée-Morvan D, Biver N, Crovisier J, Gautier D (1998) A Determination of the $\mathrm{HDO} / \mathrm{H}_{2} \mathrm{O}$ Ratio in comet C/1995 O1 (Hale-Bopp). Science 279:842-844. https://doi.org/10. $1126 /$ science. 279.5352 .842

Miura H, Tanaka KK, Yamamoto T, Nakamoto T, Yamada J, Tsukamoto K, Nozawa J (2010) Formation of cosmic crystals in highly supersaturated silicate vapor produced by planetesimal bow shocks. Astrophys J 719:642-654. https://doi.org/10.1088/0004-637X/719/1/642

Morfill G, Spruit H, Levy EH (1993) Physical processes and conditions associated with the formation of protoplanetary disks. In: Levy EH, Lunine Jl (eds). Protostars and Planets III. University of Arizona Press, Tucson. pp 939-978. https://ui.adsabs.harvard.edu/abs/1993prpl.conf..939M/abstract

Mostefaoui S, Kita NT, Togashi S, Tachibana S, Nagahara H, Morishita Y (2002) The relative formation ages of ferromagnesian chondrules inferred from their initial aluminum26/aluminum27 ratios. Meteorit Planet Sci 37:421-438. https://doi.org/10.1111/j.1945-5100.2002.tb00825.x

Mousis O, Ronnet T, Brugger B, Ozgurel O, Pauzat F, Ellinger Y, Maggiolo R, Wurz P, Vernazza P, Lunine Jl, Luspay-Kuti A, Mandt KE, Altwegg K, Bieler A, Markovits A, Rubin M (2016) Origin of molecular oxygen in comet 67P/Churyumov-Gerasimenko. Astrophys J 823:L41. https://doi.org/10. 3847/2041-8205/823/2/L41 
Muranushi T (2010) Dust-dust collisional charging and lightning in protoplanetary discs. Mon Not R Astron Soc 401:2641-2664. https://doi. org/10.1111/j.1365-2966.2009.15848.x

Muranushi T, Akiyama E, Inutsuka S, Nomura H, Okuzumi S (2015) Development of a method for the observation of lightning in protoplanetary disks using ion lines. Astrophys J 815(2):84. https://doi.org/ 10.1088/0004-637X/815/2/84

Nagahara H (1981) Petrology of chondrules in ALH-77015 (L3) chondrite. Memoirs Natl Inst Polar Res 20:145-160. https://nipr.repo.nii.ac.jp/?action= pages_view_main\&active_action=repository_view_main_item_detail\& item_id=1210\&item_no=1\&page_id=13\&block_id=104. Special issue

Nakano Y, Hashimoto A (2020) Bubbles to Chondrites-I. Evaporation and condensation experiments, and formation of chondrules. Prog Earth Planet Sci 7:47. https://doi.org/10.1186/s40645-020-00335-1

National Research Council (1986) The earth's electrical environment. In: Consensus Study Report. The National Academies Press, Washington. p 1. https://doi.org/10.17226/898

Nelson AF, Ruffert M (2005) A proposed origin for chondrule-forming shocks in the solar nebula. In: Krot AN, Scott ERD, Reipurth B (eds). Chondrites and the Protoplanetary Disk. APS, San Francisco. pp 903-912. http://www. aspbooks.org/a/volumes/article_details/?paper_id=2436

Noguchi T, Ohashi N, Tsujimoto S, Mitsunari T, Bradley JP, Nakamura T, Toh S, Stephan T, Iwata N, Imae N (2015) Cometary dust in Antarctic ice and snow: Past and present chondritic porous micrometeorites preserved on the earth's surface. Earth Planet Sci Lett 410:1-11. https://doi.org/10.1016/j. epsl.2014.11.012

Palme H, Lodders K, Jones A (2014) Solar system abundances of the elements. In: Davis AM (ed). Treatise on Geochemistry, Vol.2, Planets, Asteroids, Comets and The Solar System, 2nd Edition. Elsevier-Pergamon, Oxford. pp 15-36. https://www.sciencedirect.com/science/article/pii/ B9780080959757001182

Parise B, Caux E, Castets A, Ceccarelli C, Loinard L, Tielens AGGM, Bacmann A, Cazaux S, Comito C, Helmich F, Kahane C, Schilke P, van Dishoeck E, V. Wakelam aAW (2005) HDO abundance in the envelope of the solar-type protostar IRAS 16293-2422. Astron Astrophys 431:547-554. https://doi. org/10.1051/0004-6361:20041899

Parise B, Simon T, Caux E, Dartois E, Ceccarelli C, Rayner J, Tielens AGGM (2003) Search for solid HDO in low-mass protostars. Astron Astrophys 410:897-904. https://doi.org/10.1051/0004-6361:20031277

Persson MV, Jørgensen JK, van Dishoeck EF (2013) Warm water deuterium fractionation in IRAS 16293-2422. Astron Astrophyscis 549:L3. https://doi. org/10.1051/0004-6361/201220638

Persson MV, Jørgensen JK, van Dishoeck EF, Harsono D (2014) The deuterium fractionation of water on solar-system scales in deeply-embedded low-mass protostars. Astron Astrophys 563:A74. https://doi.org/10.1051/ 0004-6361/201322845

Pierel JDR, Nixon CA, Lellouch E, Fletcher LN, Bjoraker GL, Achterberg RK, Bézard B, Hesman BE, Irwin PGJ, Flasar FM (2017) D/H ratios on Saturn and Jupiter from Cassini CIRS. Astrohpysical J 154(5):178. https://doi.org/10. 3847/1538-3881/aa899d

Pilipp W, Hartquist TW, Morfill GE (1992) Large electric fields in acoustic waves and the stimulation of lightning discharges. Astrophys J 387:364-371. https://doi.org/10.1086/171089

Pilipp W, Hartquist TW, Morfill GE, Levy EH (1998) Chondrule formation by lightning in the Protosolar Nebula? Astron Astrophys 331:1221-146. https://ui.adsabs.harvard.edu/abs/1998A\%26A...331..121P/abstract

Rakov VA (2013) The physics of lightning. Surv Geophys 34:701-729. https:// doi.org/10.1007/s10712-013-9230-6

Rakov VA, Uman MA (2003) Lightning: Physics and Effects. Cambridge University Press, Cambridge. https://doi.org/10.1017/CBO9781107340886

Rubin AE (2000) Petrologic, geochemical and experimental constraints on models of chondrule formation. Earth Sci Rev 50:3-27. https://doi.org/10. 1016/S0012-8252(99)00067-7

Rubin AE (2003) Chromite-plagioclase assemblages as a new shock indicator; implications for the shock and thermal histories of ordinary chondrites. Geochim Cosmochim Acta 67:2695-2709. https://doi.org/10.1016/S00167037(03)00107-8

Rubin AE (2004) Postshock annealing and postannealing shock in equilibrated ordinary chondrites: implications for the thermal and shock histories of chondritic asteroids. Geochim Cosmochim Acta 68:673-689. https://doi. org/10.1016/S0016-7037(03)00452-6
Rubin AE, Huber H, Wasson JT (2009) Possible impact-induced refractory-lithophile fractionations in EL chondrites. Geochim Cosmochim Acta 73:1523-1537. https://doi.org/10.1016/j.gca.2008.12.002

Rubin AE, Wasson JT (2011) Shock effects in "EH6" enstatite chondrites and implications for collisional heating of the $\mathrm{EH}$ and $\mathrm{EL}$ parent asteroids. Geochim Cosmochim Acta 75:3757-3780. https://doi.org/10.1016/j.gca. 2011.04 .002

Russell SS, Srinivasan G, Huss GR, Wasserburg GJ, MacPherson GJ (1996) Evidence for widespread ${ }^{26} \mathrm{Al}$ in the solar nebula and constraints for nebula time scales. Science 273:757-762. https://doi.org/10.1126/science. 273.5276 .757

Saunders C (2008) Charge separation mechanisms in clouds. Space Sci Rev 137:335-353. https://doi.org/10.1007/s11214-008-9345-0

Schulz R, Hilchenbach M, Langevin Y, Kissel J, Silen J, Briois C, Engrand C, Hornung K, Baklouti D, Bardyn A, Cottin H, Fischer H, Fray N, Godard M, Lehto H, Le Roy L, Merouane S, Orthous-Daunay FR, Paquette J, Rynö J, Siljeström S, Stenzel O, Thirkell L, Varmuza K, Zaprudin B (2015) Comet 67P/Churyumov-Gerasimenko sheds dust coat accumulated over the past four years. Nature 518:216-218. https://doi.org/10.1038/nature14159

Surville C, Mayer L, Lin DNC (2016) Dust capture and long-lived density enhancements triggered by vortices in $2 \mathrm{D}$ protoplanetary disks. Astrophys J 831(1):82. https://doi.org/10.3847/0004-637X/831/1/82

Takahashi T (1978) Riming electrification as a charge generation mechanism in thunderstorms. J Atmos Sci 35:1536-1548. https://doi.org/10.1175/15200469(1978)035<1536:REAACG > 2.0.CO;2

Takahashi T, Miyawaki K (2002) Reexamination of riming electrification in a wind tunnel. J Atmos Sci 59:1018-1025. https://doi.org/10.1175/15200469(2002)059<1018:ROREIA>2.0.CO;2

Tanga P, Babiano A, Dubrullec B, Provenzale A (1996) Forming planetesimals in vortices. Icarus 121:158-170. https://doi.org/10.1006/icar.1996.0076

Taquet V, Furuya K, Walsh C, van Dishoeck EF (2016) A primordial origin for molecular oxygen in comets: a chemical kinetics study of the formation and survival of $\mathrm{O}_{2}$ ice from clouds to discs. Mon Not R Astron Soc 462:S99-S115. https://doi.org/10.1093/mnras/stw2176

Taquet V, López-Sepulcre A, Ceccarelli C, Neri R, Kahane C, Coutens A, Vastel C (2013) Water deuterium fractionation in the inner regions of two solar-type protostars. Astrophys J Lett 768(2):L29. https://doi.org/10.1088/2041-8205/ 768/2/L29

Tielens AGGM (2013) The molecular universe. Rev Mod Phys 85:1021-1081. https://doi.org/10.1103/RevModPhys.85.1021

Tomeoka K, Buseck PR (1985) Indicators of aqueous alteration in CM carbonaceous chondrites: Microtextures of a layered mineral containing Fe, S, O and Ni. Geochim Cosmochim Acta 49:2149-2163. https://doi.org/ 10.1016/0016-7037(85)90073-0

Urey HC, Craig H (1953) The composition of the stone meteorites and the origin of the meteorites. Geochim Cosmochim Acta 4:36-82. https://doi. org/10.1016/0016-7037(53)90064-7

Villanueva GL, Mumma MJ, Bonev BP, DiSanti MA, Gibb EL, Böhnhardt H, Lippi M (2009) A sensitive search for deuterated water in comet 8P/Tuttle. Astrophys J Lett 690:L5. https://doi.org/10.1088/0004-637X/690/1/L5

Visser R, Jørgensen JK, Kristensen LE, van Dishoeck EF, Bergin EA (2013) Hot water in the inner 100 au of the class 0 protostar NGC 1333 IRAS2A. Astrophys J 769(1):19. https://doi.org/10.1088/0004-637X/769/1/19

Walsh KJ (2018) Rubble pile asteroids. Annu Rev Astron Astrophys 56:593-624. https://doi.org/10.1146/annurev-astro-081817-052013

Walsh C, Nomura H, van Dishoeck E (2015) The molecular composition of the planet-forming regions of protoplanetary disks across the luminosity regime. Astron Astrophys 582:A88. https://doi.org/10.1051/0004-6361/ 201526751

Wasilewski P, Dickinson T (2000) Aspects of the validation of magnetic remanence in meteorites. Meteorit Planet Sci 35:537-544. https://doi.org/ 10.1111/j.1945-5100.2000.tb01434.x

Wasserburg GJ, Lee T, Papanastassiou DA (1977) Correlated O and Mg isotopic anomalies in Allende Inclusions: II, Magnesium. Geophys Res Lett 4:299-302. https://doi.org/10.1029/GL004i007p00299

Wasserburgm GJ, Wimpenny J, Yin Q-Z (2012) Mg isotopic heterogeneity, Al-Mg isochrons, and canonical ${ }^{26} \mathrm{Al} /{ }^{27} \mathrm{Al}$ in the early solar system. Meteorit Planet Sci 47:1980-1997. https://doi.org/10.1111/maps.12014

Weidenschilling SJ (1977) Aerodynamics of solid bodies in the solar nebula. Mon Not R Astron Soc 180:57-70. http://adsabs.harvard.edu/abs/ 1977MNRAS.180...57W 
Whipple FL (1966) Chondrules: Suggestion concerning the origin. Science 153:54-56. https://doi.org/10.1126/science.153.3731.54

Williams JP, Cieza LA (2011) Protoplanetary disks and their evolution. Annu Rev Astron Astrophys 49:67-117. https://doi.org/10.1146/annurev-astro081710-102548

Wood JA, Hashimoto A (1993) Mineral equilibrium in fractionated nebular systems. Geochim Cosmochim Acta 57:2377-2388. https://doi.org/10. 1016/0016-7037(93)90575-H

Yanai K, Kojima H, Haramura H (1995) Catalog of the Antarctic Meteorites. Memoirs Natl Inst Polar Res. Special issue(Hirasawa T, ed.). Natl Inst Polar Res, Tokyo

Zinner E (1998) Stellar nucleosynthesis and the isotopic composition of presolar grains from primitive meteorites. Ann Rev Earth Planet Sci 26:147-188. https://doi.org/10.1146/annurev.earth.26.1.147

\section{Publisher's Note}

Springer Nature remains neutral with regard to jurisdictional claims in published maps and institutional affiliations.

\section{Submit your manuscript to a SpringerOpen ${ }^{\circ}$ journal and benefit from:}

- Convenient online submission

- Rigorous peer review

- Open access: articles freely available online

- High visibility within the field

- Retaining the copyright to your article

Submit your next manuscript at $>$ springeropen.com 